\title{
Variationsprinzipien bei natürlichen Eigenwertproblemen
}

\author{
von W. Dück (Berlin)
}

Auf die Bedeutung der natürlichen Eigenwertprobleme für die Mechanik haben Stiefel und Ziegler [10] hingewiesen. Eine vollständige Theorie der natürlichen Eigenwertprobleme, bei denen in den restlichen Randbedingungen der Eigenparameter linear auftreten darf, wurde von Lehmann [8] - man beachte auch [9] - entwickelt. Dabei verwendet Lehmann den Übergang zur Integraldarstellung. Bis heute ist aber noch nicht untersucht worden, ob die von Kamke [5], [6] (') entwickelte Eigenwerttheorie, die voraussetzt, daß die Randbedingungen vom Eigenparameter frei sind, sich nicht auch auf natürliche Eigenwertprobleme ausdehnen läßt. In Anlehnung an die Arbeiten von Lehmann wurden bereits von Dück [2], [3], [4] einige Erweiterungen der Kamkeschen Eigenwerttheorie angegeben. Es wurden dort aber noch nicht natürliche Eigenwertprobleme erfaßt. Grundlage der Beschreibung der natürlichen Eigenwertprobleme ist eine natürliche Produktbildung, die wie bei Lehmann eingenführt, deren Begründung aber im folgenden nochmals ausführlich erläutert wird. Voraussetzungen und Bezeichnungen werden so gegeben, daß eine möglichst einfache Übertragung des Kamkeschen Gedankens möglich ist, soweit nicht die Rücksichtnahme auf andere Arbeiten eine Abweichung davon sinvoll erscheinen läßt. Zur Abkürzung der Beweise ist vielfach auf die Arbeiten von Kamke verwiesen worden. Nach Angabe einiger grundlegender Eigenschaften natürlicher Eigenwertprobleme werden die Variationsprinzipien von Rayleigh und Courant und Vergleichungssätze bewiesen. Wir werden erkennen, daß die von Kamke verwendete Beschreibungsweise von Eigenwertaufgaben in ihren Grundzügen so leistungsfähig ist, daß sie auch die Behandlung von natürlichen Eigenwertproblemen gestattet.

1. Problemstellung. Betrachtet wird die Differentialgleichung

$$
M y-\lambda N y=0
$$

(1) Wegen der großen Wichtigkeit dieser Arbeiten für die folgenden Ausführungen wird künftig auf [5] durch K II und auf [6] durch $K$ III verwiesen. 
mit den linearen selbstadjungierten gewöhnlichen Differentialausdrücken

$$
\begin{aligned}
& M y=\sum_{i=0}^{m}(-1)^{i}\left\{f_{i}(x) y^{(i)}(x)\right\}^{(i)}, \\
& N y=\sum_{i=0}^{n}(-1)^{i}\left\{g_{i}(x) y^{(i)}(x)\right\}^{(i)} .
\end{aligned}
$$

$f_{l}(x)$ und $g_{i}(x)$ seien gegebene, reelle, $i$-mal im Grundintervall $a \leqslant x \leqslant b$ stetig differenzierbare Funktionen und es möge

gelten.

$$
f_{m}(x) \neq 0, \quad g_{n}(x) \neq 0, \quad 0 \leqslant n<m
$$

Zur Differentialgleichung treten $2 m$ voneinander linear unabhängige, lineare homogene Randbedingungen hinzu, und zwar $k$ wesentliche Randbedingungen

$$
\sum_{i=0}^{m-1}\left\{a_{i j}^{\prime \prime i} y^{(i)}(a)+\beta_{i j}^{W \cdot} y^{(i)}(b)\right\}=0, \quad j=1, \ldots, k,
$$

die nur Randableitungen bis zur Ordnung $m-1$ enthalten, und $2 m-k$ restliche Randbedingungen

$$
\begin{array}{r}
\sum_{i=0}^{2 m-1}\left\{\alpha_{i j}^{M} y^{(i)}(a)+\beta_{i j}^{M} y^{(i)}(b)\right\}=\lambda \sum_{i=0}^{2 m-1}\left\{a_{i j}^{N} y^{(i)}(a)+\beta_{i j}^{\mathrm{V}} y^{(i)}(b)\right\}, \\
j=1, \ldots, 2 m-k,
\end{array}
$$

in die der Eigenparameter $\lambda$ linear eingehen darf. Dabei soll in bekannter Weise (2) alle Randbedingungen umfassen, in denen nur Randableitungen bis zur Ordnung $m-1$ auftreten, und es darf auch nicht durch Linearkombination der Randbedingungen möglich sein, weitere der Gestalt (2) zu gewinnen. Die Konstanten $\alpha_{i j}^{W V}, \alpha_{i j}^{Y}, \alpha_{i j}^{N}, \beta_{i j}^{T}, \beta_{i j}^{Y I}, \beta_{i j}^{N}$ in (2), (3) sollen gegeben und reell sein.

Im folgenden schreiben wir für die wesentlichen Randbedingungen kurz

$$
W_{j}(y)=0, \quad j=1, \ldots, k,
$$

und für die restlichen

$$
R_{j}^{M}(y)=\lambda R_{j}^{N}(y), \quad j=1, \ldots, 2 m-k .
$$

Wir werden künftig (4), (5) natiilliche Randbedingungen nennen.

Die von Kamke gemachte Voraussetzung

$$
R_{j}^{N}(y)=0, \quad j=1, \ldots, 2 m-k
$$

beschneidet die Problemklasse sehr. Wir sprechen dann von Kamkeschen Randbedingungen. 
Im Falle $k=2 m$ sind alle Randbedingungen wesentlich, so daß diese Aufgaben von der Kamkeschen Eigenwerttheorie erfaßt werden. Es ist auch $k=0$ zulässig; dann liegen nur restliche Randbedingungen vor.

2. Erklärung einiger Funktionenräume. Raum der zulässigen Funktionen $Z$ : Enthält alle zulässigen Funktionen und die im Grundintervall identisch verschwindende Funktion.

Eine zulässige Funktion soll $(m-1)$-mal stetig und stückweise stetig sogar $m$-mal in $a \leqslant x \leqslant b$ differenzierbar sein, den wesentlichen Randbedingungen genügen und im Grundintervall nicht identisch verschwinden.

Raum der streng zulässigen Frunktionen $\bar{Z}$ : Umfaßt alle streng zulässigen Funktionen und die im Grundintervall identisch verschwindende Funktion.

Eine streng zulässige Funktion ist eine zulässige Funktion, die in $a \leqslant x \leqslant b$ sogar $2 m$-mal stetig differenzierbar ist.

Raum der verallgemeinerten zulässigen Funktionen $Z^{*}\left({ }^{2}\right)$ : Er enthält alle verallgemeinerten zulässigen Funktionen und die identisch verschwindende Funktion.

Unter einer verallgemeinerten zulässigen Funktion soll eine $(m-1)$-mal im Grundinterval stetig differenzierbare Funktion verstanden werden, die den wesentlichen Randbedingungen genügt und in $a \leqslant x \leqslant b$ nicht identisch verschwindet.

Raum der Vergleichsfunktionen $V$ : Dieser Raum wird nur für Aufgaben mit Kamkeschen Randbedingungen erklärt. Er umfaßt sämtliche Vergleichsfunktionen und die identisch verschwindende Funktion.

Eine Vergleichsfunktion soll dabei $2 m$-mal in $a \leqslant x \leqslant b$ stetig differenzierbar sein, den wesentlichen und den restlichen Randbedingungen genügen und nicht identisch verschwinden.

Sämtliche eingeführten Räume sind linear.

\section{Einführung natürlicher Produktbildungen.}

3.1. Bezeichnungen. Von den $2 m$ Randableitungen

$$
u^{(i)}(a), \quad u^{(i)}(b), \quad i=0,1, \ldots, m-1,
$$

einer zulässigen Funktion $u(x)$ können mittels der wesentlichen Randbedingungen (4) $k$ Randableitungen durch die übrigen $2 m-k$ ausgedrückt werden. Diese $2 m-k$ Randableitungen heißen freie Randableitungen, sie können offensichtlich beliebig vorgegeben werden, während die restlichen $k$ Randableitungen dann durch die wesentlichen Randbedingungen bestimmt sind. Die freien Randableitungen fassen wir als Komponenten eines Spaltenvektors $\boldsymbol{x}(u)$ auf, der freier Randvektor genannt werden soll.

(2) Der Raum Z* wird in dieser Arbeit keine Rolle spielen. Mit Rücksicht auf die Betrachtungen in einer späteren Veröffentlichung wird bereits hier die Definition dieses Raumes angegeben. 
Weiterhin erklären wir zwei Zeilenvektoren $\mathfrak{R}^{M}(u)$ und $\mathfrak{R}^{N}(u)$, die durch die Randoperatoren der restlichen Randbedingungen (5) bestimmt sind:

$$
\begin{aligned}
& \Re^{M}(u)=\left(R_{1}^{M}(u), \ldots, R_{2 m-k}^{M I}(u)\right), \\
& \mathfrak{R}^{N}(u)=\left(R_{1}^{N}(u), \ldots, R_{\beth_{m-k}^{N}}^{N}(u)\right) .
\end{aligned}
$$

Es besteht zunächst Freiheit in der Wahl der Reihenfolge der Komponenten der Vektoren $\mathfrak{x}(u)$ und $\mathfrak{R}^{M}(u)$ und auch der Vorzeichen von $R_{j}^{M}(u)$. Diese Freiheit wird eingeschränkt, wenn die unten gemachten Voraussetzungen erfüllt werden sollen. Man beachte dazu auch die Bemerkungen in 3.6.

Zur Vereinfachung der Schreibweise führen wir weiterhin die Abkürzungen

$$
\langle u, v\rangle=\int_{a}^{b} u(x) M v(x) d x, \quad[u, v]=\int_{a}^{b} u(x) N v(x) d x
$$

ein. Dabei sollen die Funktionen $u(x), v(x)$ die für diese Verknüpfungen notwendigen Stetigkeits- und Differenzierbarkeitsvoraussetzungen erfüllen.

3.2. Das Problem der Einführung von skalaren Verknulpfungen in $Z$. Bei Aufgaben mit Kamkeschen Randbedingungen werden $\langle u, v\rangle$ und $[u, v]$ als skalare Verknüpfungen im Raum der Vergleichsfunktionen verwendet. Denn die bei selbstadjungierten Aufgaben giiltigen Symmetrierelationen

$$
\langle u, v\rangle=\langle v, u\rangle, \quad[u, v]=[v, u]
$$

machen sie zu geeigneten Verknüpfungen in $V$. Aber schon für die Betrachtung von Eigenwertproblemen mit Kamkeschen Randbedingungen in $Z$ sind diese Bildungen, wie Dück [3], [4] gezeigt hat, nicht mehr verwendbar. Es ist leicht zu erkennen, daß auch bei natürlichen Eigenwertproblemen in $\bar{Z}\langle u, v\rangle$ und $[u, v]$ keine geeigneten skalaren Verkniipfungen sind, weil die Symmetrieforderung ( 7 ) in $\bar{Z}$ keine sinnvolle Voraussetzung über das Problem sein kann. Der Schlüssel zur Angabe von skalaren Verknüpfungen in $Z$ liegt in der Untersuchung der Dirichletschen Restteile.

3.3. Umformung der Dirichlet schen Restteile. In der Dirichletschen Formel

$$
\begin{aligned}
& \langle u, v\rangle=\int_{a}^{b} \sum_{i=0}^{m} f_{i}(x) u^{(i)}(x) v^{(i)}(x) d x+\left.R(u, v)\right|_{a} ^{b}, \\
& {[u, v]=\int_{a}^{b} \sum_{i=0}^{n} g_{i}(x) u^{(i)}(x) v^{(i)}(x) d x+S(u, v)_{\mid a}^{b}}
\end{aligned}
$$


sind $\left.R(u, v)\right|_{a} ^{b}$ und $\left.S(u, v)\right|_{a} ^{b}$ die Dirichletschen Restteile. Entnehmen wir $u(x)$ aus $Z, v(x)$ aus $\bar{Z}$ und tragen wir in den Dirichletschen Restteilen die wesentlichen Randbedingungen ein, entstehen. Restteile, die wir mit $\left.\bar{R}(u, v)\right|_{a} ^{b}$ und $\left.\bar{S}(u, v)\right|_{a} ^{b}$ bezeichnen wollen. Sie sind Bilinearformen in den freien Randableitungen von $u(x)$ und $v(x)$ und den höheren Ableitungen von $v(x)$

$$
\left.R(u, v)\right|_{a} ^{b}=\left.\bar{R}(u, v)\right|_{a} ^{b},\left.\quad S(u, v)\right|_{a} ^{b}=\left.\bar{S}(u, v)\right|_{a} ^{b}, \quad u \in Z, v \in \bar{Z} .
$$

Dann hängen auch die Ausdrïcke

$$
\left.\bar{R}(u, v)\right|_{a} ^{b}-\mathfrak{R}^{M}(v) \mathfrak{x}(u) \quad \text { und }\left.\quad \bar{S}(u, v)\right|_{a} ^{b}-\mathfrak{R}^{N}(v) \mathfrak{x}(u)
$$

von den freien Randableitungen von $u(x), v(x)$ und den höheren Ableitungen von $v(x)$ ab. Wir können sie so aufteilen, daß wir die nur von den freien Randableitungen der Funktionen $u(x), v(x)$ abhängigen Glieder in einer ersten und den Rest in einer zweiten Bilinearform vereinigen. Wir schreiben

$$
\begin{aligned}
\left.\bar{R}(u, v)\right|_{a} ^{b}-\mathfrak{R}^{M}(v) \mathfrak{x}(u) & =\mathfrak{x}^{\prime}(u) \mathfrak{M}_{\mathfrak{x}}(v)+\left.R^{*}(u, v)\right|_{a} ^{b}\left(^{3}\right), \quad u \in Z, v \in \bar{Z} . \\
\left.\bar{S}(u, v)\right|_{a} ^{b}-\mathfrak{R}^{N}(v) \mathfrak{x}(u) & =\mathfrak{x}^{\prime}(u) \mathfrak{M}_{\mathfrak{x}}(v)+\left.S^{*}(u, v)\right|_{a} ^{b},
\end{aligned}
$$

$\mathfrak{x}^{\prime}(u) \mathfrak{M} \mathfrak{x}(v)$ und $\mathfrak{x}^{\prime}(u) \mathfrak{M}_{\mathfrak{x}}(v)$ nennen wir die durch die Operatoren $M$ und $N$ vermittelten natürlichen Restteile. Sie hängen nur von den freien Randableitungen von $u(x)$ und $v(x)$ ab. $\left.R^{*}(u, v)\right|_{a} ^{b}$ und $\left.S^{*}(u, v)\right|_{a} ^{b}$ sind Bilinearformen in den freien Randableitungen von $u(x)$ und den höheren Ableitungen von $v(x)$.

\subsection{Erklärung selbstadjungierter natürlicher Eigenwert-} probleme. Wir orientieren uns zunächst am Sonderfall der Aufgaben mit Kamkeschen Randbedingungen. Wählen wir $v(x)$ aus $V$, ist $\mathfrak{R}^{M}(v)$ gleich dem Nullvektor, und zusammen mit (6) erhalten wir

$$
\begin{aligned}
&\left.\bar{R}(u, v)\right|_{a} ^{b}=\mathfrak{x}^{\prime}(u) \mathfrak{M}_{\mathfrak{x}}(v)+\left.R^{*}(u, v)\right|_{a} ^{b}, \quad u \in Z, \quad v \in V . \\
&\left.\bar{S}(u, v)\right|_{a} ^{b}=x^{\prime}(u) \mathfrak{M}_{\mathfrak{x}}(v)+\left.S^{*}(u, v)\right|_{a} ^{b},
\end{aligned}
$$

Ist die Aufgabe selbstadjungiert, gilt also (7) in $V$, können, wie Kamke [i] zeigt, in den Dirichletschen Restteilen alle Ableitungen der Ordnung $\geqslant m$ beseitigt werden:

$$
\begin{aligned}
& \left.R(u, v)\right|_{a} ^{b}=x^{\prime}(u) \overline{\mathfrak{M}} x(v), \quad u \in Z, v \in V . \\
& \left.S(u, v)\right|_{a} ^{b}=x^{\prime}(u) \overline{\mathfrak{N}} \mathfrak{x}(v),
\end{aligned}
$$

(a) $\mathbf{x}^{\prime}(u)$ bezeichnet den $\mathrm{zu} x(u)$ transponierten Vektor. 
$\mathfrak{x}^{\prime}(u) \overline{\mathfrak{M}}_{\mathfrak{x}}(v)$ und $\mathfrak{x}^{\prime}(u) \overline{\mathfrak{N}} \mathfrak{x}(v)$ heißen auch reduzierte Dirichletsche Restteile. Damit folgt aus (9), (10) bei Beachtung von (8)

$$
\begin{aligned}
&\left.R^{*}(u, v)\right|_{a} ^{b}=\mathfrak{x}^{\prime}(u) \overline{\mathfrak{M}} \mathfrak{x}(v)-\mathfrak{x}^{\prime}(u) \mathfrak{M} \mathfrak{X}(v), u \in Z, v \in V . \\
&\left.S^{*}(u, v)\right|_{a} ^{b}=\mathfrak{x}^{\prime}(u) \overline{\mathfrak{N}} \mathfrak{x}(v)-\mathfrak{x}^{\prime}(u) \mathfrak{N} \mathfrak{x}(v),
\end{aligned}
$$

Die rechten Seiten dieser Gleichungen hängen nur von den freien Randableitungen von $u(x), v(x)$ ab, also muß das auch für $\left.R^{*}(u, v)\right|_{a} ^{b}$ und $\left.S^{*}(u, v)\right|_{a} ^{b}$ gelten, was nicht im Einklang mit ihrer obigen Bildung steht. Bei Aufgaben mit Kamkeschen Randbedingungen folgt aus der Symmetrieforderung

$$
\left.R^{*}(u, v)\right|_{a} ^{b}=\left.S^{*}(u, v)\right|_{a} ^{b}=0, \quad u \in Z, v \in V
$$

und damit auch, daß die natürlichen Restteile mit den reduzierten Dirichletschen Restteilen übereinstimmen:

$$
\begin{aligned}
& \mathfrak{x}^{\prime}(u) \overline{\mathfrak{M}}_{\mathfrak{x}}(v)=\mathfrak{x}^{\prime}(u) \mathfrak{M}_{\mathfrak{x}}(v), \quad u \in Z, v \in V . \\
& \mathfrak{x}^{\prime}(u) \overline{\mathfrak{N}}_{\boldsymbol{x}}(v)=\mathfrak{x}^{\prime}(u) \mathfrak{N} \boldsymbol{x}(v),
\end{aligned}
$$

Weiterhin führt die Voraussetzung der Selbstadjungiertheit bei diesen Aufgaben zur Symmetrie der Matrizen $\overline{\mathfrak{M}}$ und $\overrightarrow{\mathfrak{N}}$.

Es ist jetzt, sehr einfach, eine sinnvolle Definition eines selbstadjungierten natürlichen Eigenwertproblems zu geben. Wir wollen die Aufgabe (1), (2), (3) selbstadjungiert nennen, wenn

1) $\mathfrak{M}$ und $\mathfrak{R}$ symmetrische Matrizen bezeichnen,

2) $\left.R^{*}(u, v)\right|_{a} ^{b}=\left.S^{*}(u, v)\right|_{a} ^{b}=0$ für jedes $u \in Z, v \in \bar{Z}$ gilt.

Dann ist

$$
\begin{aligned}
& \left.\bar{R}(u, v)\right|_{a} ^{b}-\mathfrak{R}^{. I}(v) \mathfrak{x}(u) \doteq \mathfrak{x}^{\prime}(u) \mathfrak{M} \mathfrak{x}(v), \quad u \in Z, v \in \bar{Z} . \\
& \left.\bar{S}(u, v)\right|_{a} ^{b}-\mathfrak{R}^{N}(v) \mathfrak{x}(u)=\mathfrak{x}^{\prime}(u) \mathfrak{N} \mathfrak{x}(v),
\end{aligned}
$$

3.5. Definition nati̊rlicher Produktbildungen. Unabhängig von der Herleitung definieren wir nun für selbstadjungierte Aufgaben zwei Verknüpfungen in $Z$, die natürliche Produktbildungen heißen sollen, durch die Gleichungen

$$
\begin{aligned}
& (u, v)_{M}=\sum_{i=0}^{m} \int_{a}^{b} f_{i}(x) u^{(i)}(x) v^{(i)}(x) d x+\mathbf{x}^{\prime}(u) \mathfrak{M} \mathbf{x}(v), \quad u, v \in Z \\
& (u, v)_{N}=\sum_{i=0}^{n} \int_{a}^{b} g_{i}(x) u^{(i)}(x) v^{(i)}(x) d x+\mathbf{x}^{\prime}(u) \mathfrak{M}(v), \quad u, v \in Z^{*} .
\end{aligned}
$$


Dabei erkennt man, daß wegen der Voraussetzung $n<m$ (14) sogar in $Z^{*}$ einen Sinn hat. Bei selbstadjungierten natürlichen Eigenwertproblemen sind diese Bildungen symmetrisch in $u(x)$ und $v(x)$

$$
\begin{aligned}
(u, v)_{M}=(v, u)_{M}, & u, v \in Z, \\
(u, v)_{X}=(v, u)_{N}, & u, v \in Z^{*} .
\end{aligned}
$$

Wegen der Betrachtungen in 3.4. gehen die natürlichen Produktbildungen für Aufgaben mit Kamkeschen Randbedingungen in die skalaren Verknüpfungen über, die bereits von Dück in [2], [3], [4] verwendet wurden.

Aus der Dirichletschen Formel folgen, zusammen mit (8), (11), (12) und den Definitionsgleichungen der natürlichen Produktbildungen, für selbstadjungierte Aufgaben die fundamentalen Beziehungen

$$
\begin{aligned}
& \langle u, v\rangle=(u, v)_{M}+\mathfrak{R}^{M}(v) \mathfrak{x}(u), \quad u \in Z, v \in \bar{Z}, \\
& {[u, v]=(u, v)_{N}+\Re^{N}(v) \mathfrak{x}(u), \quad u \in Z^{*}, v \in \bar{Z} .}
\end{aligned}
$$

3.6. Beispiele und wichtige Bemerkungen zur Angabe natürlicher Produktbildungen. Wir betrachten zunächst ein sehr einfaches Beispiel. Vorgelegt seien die Differentialgleichung

$$
-u^{\prime \prime}-\lambda u=\mathbf{0},
$$

die wesentliche Randbedingung

$$
u(1)=0
$$

und die restliche Randbedingung

$$
u^{\prime}(0)=-\lambda u(0) .
$$

Die Vektoren $x(u), \mathfrak{R}^{u}(u), \mathfrak{R}^{v}(u)$ sind hier eindimensional, so daß keine Freiheit in der Wahl der Reihenfolge ihrer Komponenten auftritt. Wir setzen

$$
\mathfrak{R}^{M}(u)=\left(u^{\prime}(0)\right), \quad \Re^{N}(u)=(-u(0)) .
$$

Von den Randableitungen $u(0), u(1)$ einer zulässigen Funktion bis zur Ordnung $m-1$ ist $u(1)$ durch die wesentliche Randbedingung bestimmt. Also ist $u(0)$ die freie Randbleitung und

Es gilt

$$
\mathfrak{x}(u)=(u(0)) .
$$

$$
\begin{array}{ll}
\left.R(u, v)\right|_{0} ^{1}=-\left.u(x) v^{\prime}(x)\right|_{0} ^{1}, & \left.S(u, v)\right|_{0} ^{1}=0, \\
\left.\bar{R}(u, v)\right|_{0} ^{1}=u(0) v^{\prime}(0)=\Re^{M}(v) \mathbf{x}(u), & \left.\bar{S}(u, v)\right|_{0} ^{1}=\mathbf{0} .
\end{array}
$$

Damit folgt aus (11), (12) für die natürlichen Restteile

$$
\mathfrak{x}^{\prime}(u) \mathfrak{M}_{\mathfrak{x}}(v)=0, \quad \mathfrak{x}^{\prime}(u) \mathfrak{N} \mathfrak{x}(v)=-\mathfrak{R}^{N}(v) \mathfrak{x}(u)=v(0) u(0) .
$$


Die natürlichen Produktbildungen lauten

$$
(u, v)_{M}=\int_{0}^{1} u^{\prime}(x) v^{\prime}(x) d x, \quad \cdot(u, v)_{N}=\int_{0}^{1} u(x) v(x) d x+u(0) v(0) .
$$

Insbesondere ergibt sich auch die Selbstadjungiertheit des natürlichen Eigenwertproblems.

Wir behandeln noch ein zweites Beispiel, und zwar die Differentialgleichung

$$
u^{\mathbf{I V}}=\lambda\left(-u^{\prime \prime}+u\right)
$$

mit den wesentlichen Randbedingungen

$$
u(0)=0, \quad u^{\prime}(1)=-u(1)
$$

und den restlichen Randbedingungen

und setzen

$$
u^{\prime \prime}(0)=0, \quad u^{\prime \prime \prime}(1)+u^{\prime \prime}(1)=\lambda u(1)
$$

$$
\mathfrak{R}^{M}(u)=\left(u^{\prime \prime}(0), u^{\prime \prime \prime}(1)+u^{\prime \prime}(1)\right), \cdot \mathfrak{R}^{N}(u)=(0, u(1)) .
$$

Von den Randableitungen $u(0), u(1), u^{\prime}(0), u^{\prime}(1)$ einer zulässigen Funktion können $u(0)$ und $u^{\prime}(1)$ als durch die wesentlichen Randbedingungen bestimmt angesehen werden, so daß $u(1), u^{\prime}(0)$ die freien Randableitungen sind. Wir wählen

$$
\mathfrak{x}(u)=\left(u^{\prime}(\mathbf{0}), u(\mathbf{1})\right) .
$$

Dann ergibt sich

$$
\begin{aligned}
& \left.R(u, v)\right|_{0} ^{1}=\left.u(x) v^{\prime \prime \prime}(x)\right|_{0} ^{1}-\left.u^{\prime}(x) v^{\prime \prime}(x)\right|_{0} ^{1}, \\
& \left.S(u, v)\right|_{0} ^{1}=-\left.u(x) v^{\prime}(x)\right|_{0} ^{1}, \\
& \left.\bar{R}(u, v)\right|_{0} ^{1}=u(\mathbf{1}) v^{\prime \prime \prime}(\mathbf{1})+u(1) v^{\prime \prime}(1)+u^{\prime}(0) v^{\prime \prime}(0)=\mathfrak{R}^{M}(v) \mathfrak{x}(u), \\
& \left.\bar{S}(u, v)\right|_{0} ^{1}=u(1) v(\mathbf{1})=\mathfrak{R}^{N}(v) \mathfrak{x}(u) .
\end{aligned}
$$

Die natürlichen Restteile müssen somit verschwinden.

Hätten wir jedoch den freien Randvektor in der Form

angesetzt, würden wir

$$
\boldsymbol{x}(u)=\left(u(\mathbf{1}), u^{\prime}(\mathbf{0})\right)
$$

$$
\begin{aligned}
\left.\bar{R}(u, v)\right|_{0} ^{1}-\Re^{M}(v) \mathfrak{x}(u) & =\left\{v^{\prime \prime \prime}(1)+v^{\prime \prime}(1)\right\}\left\{u(1)-u^{\prime}(0)\right\}+v^{\prime \prime}(0)\left\{u^{\prime}(0)-u(1)\right\} \\
& =\left.R^{*}(u, v)\right|_{0} ^{1}
\end{aligned}
$$

und einen entsprechenden Ausdruck für $\left.\mathcal{S}^{*}(u, v)\right|_{0} ^{1}$ finden. Hier erkennen wir sofort, daß eine Vertauschung der Reihenfolge der Komponenten von $x(u)$ zum Verschwinden der Restteile $\left.R^{*}(u, v)\right|_{0} ^{1}$ und $\left.S^{*}(u, v)\right|_{0} ^{1}$ führt. 
Ähnliche Effekte würden sich bei der Vertauschung der Reihenfolge der Komponenten bei $\mathfrak{R}^{M}(u)$ ergeben. Außerdem tritt noch eine gewisse Freiheit in der Wahl der Vorzeichen der Komponenten von $\mathfrak{R}^{M}(u)$ auf. So hätten wir z. B.

$$
\mathfrak{R}^{M}(u)=\left(u^{\prime \prime}(0),-u^{\prime \prime \prime}(1)-u^{\prime \prime}(1)\right), \quad \Re^{N}(u)=(0,-u(1))
$$

wählen können. Das würde mit dem freien Randvektor (19)

$$
\left.\bar{R}(u, v)\right|_{0} ^{1}-\Re^{M}(v) \geq(u)=2 u(1)\left\{v^{\prime \prime \prime}(1)+v^{\prime \prime}(1)\right\}=\left.R^{*}(u, v)\right|_{0} ^{1}
$$

ergeben. Es tritt ein Rest $\left.R^{*}(u, v)\right|_{0} ^{1}$ auf, der das doppelte Produkt einer Komponente von $\mathfrak{R}^{M}(v)$ und $\mathfrak{x}(u)$ ist. Diese Vorzeichenfreiheit führt zu $2^{2 m-k}$ Möglichkeiten in der Wahl der Randoperatoren.

Bei Problemen mit Kamkeschen Randbedingungen ist, was bei natürlichen Eigenwertproblemen noch hinzukommt, die Wahl der Vorzeichen der Operatoren offen; denn die Aufgaben $M y-\lambda N y=0$ und $(-M) y-\lambda(-N) y=0$ sind äquivalent. Diese Vorzeichen werden aber durch gewisse Definitheitsvoraussetznugen praktisch festgelegt. Die bei natürlichen Eigenwertproblemen auftretende wesentlich größere Freiheit dürfte in der Praxis kaum zu Schwierigkeiten führen, so daß sich eine über die gemachten Andeutungen hinausgehende Untersuchung erübrigen dürfte. Die $\mid$ im folgenden einzuführenden Definitheitsvoraussetzungen bewirken eine weitere Reduktion dieser Freiheiten.

4. Definitheitsvoraussetzungen. Wir nennen ein selbstadjungiertes natürliches Eigenwertproblem eigentlich semidefinit in $Z$, wenn

$$
(u, u)_{M} \geqslant 0, \quad u \in Z
$$

gilt und falls für ein $u \in Z, u \neq 0,(u, u)_{M}=0$ ist, soll

$$
(u, u)_{N} \neq 0
$$

sein und für all diese $u(x)$ sogar ein festes Vorzeichen haben.

Ein selbstadjungiertes natürliches Eigenwertproblem wird eigentlich definit in $Z$ genannt, sobald

ist, definit, wenn

$$
(u, u)_{M}>0, \quad u \in Z, u \neq 0
$$

$$
(u, u)_{11}>0, \quad(u, u)_{N} \geqslant 0, \quad u \in Z, u \neq 0
$$

gilt und schießlich volldefinit im Falle

$$
(u, u)_{M}>0, \quad(u, u)_{N}>0, \quad u \in Z, u \neq 0 .
$$

Es sollen die entsprechenden Definitheitsdefinitionen in $\bar{Z}$ gegeben werden, wenn die Elemente aus $\bar{Z}$ entnommen werden. 


\section{tionen. \\ 5. Erste Aussagen uber die Eigenwerte und Eigenfunk-}

5.1. Erste Folgerungen aus den Definitheitsdefinitionen. Wir stellen hier einige Sätze zusammen, die sich unmittelbar durch Ausnutzung der Definitheitsvoraussetzungen ergeben.

SATZ 1. $\lambda=0$ ist kein Eigenwert einer in $\bar{Z}$ eigentlich definiten Aufgabe.

Beweis. Wäre $\lambda=0$ Eigenwert und $y(x)$ eine zugehörige Eigenfunktion, müßte

$$
M y=0, \quad R_{j}^{M}(y)=0, \quad j=1, \ldots, 2 m-k,
$$

gelten. Daraus würde $\langle y, y\rangle=0$ und mit (17) unter Berücksichtigung der restlichen Randbedingungen $(y, y)_{M}=0$ folgen. Das ist ein Widerspruch gegen die Voraussetzung der eigentlichen Definitheit.

SATZ 2. Ist für eine streng zulässige Funktion $u(x)$ einer in $\bar{Z}$ eigentlich semidefiniten Aufgabe

$$
(u, u)_{M}=\mathbf{0},
$$

so ist $\lambda=0$ Eigenwert und $u(x)$ eine zugehörige Eigenfunktion.

Beweis. Bezeichnet $v(x)$ eine beliebige Funktion aus $\bar{Z}$ und $\varepsilon$ eine beliebige Zahl, ist bei in $\bar{Z}$ eigentlich semidefiniten Aufgaben

$$
(u+\varepsilon v, u+\varepsilon v)_{M} \geqslant 0 \text {. }
$$

Daraus folgt mit (15), (20)

$$
2 \varepsilon(u, v)_{M}+\varepsilon^{2}(v, v)_{M} \geqslant 0 .
$$

$|\varepsilon|$ kann beliebig klein und das Vorzeichen von $\varepsilon$ beliebig gewählt werden. Daher muß

$$
(v, u)_{M}=0 \quad \text { für alle } v \in \bar{Z}
$$

sein. Wählen wir $v \in \bar{Z}$ so, daß $x(v)=0$ ist, aber sonst beliebig, folgt aus $(17)\langle v, u\rangle=0$ und daraus leicht $M u=0$. Dann muß

und damit

$$
\langle v, u\rangle=0 \quad \text { für alle } v \in \bar{Z}
$$

$$
\Re^{M}(u) x(v)=0 \quad \text { für alle } v \in \bar{Z},
$$

also für beliebige Vektoren $\boldsymbol{x}(v)$, gelten. Daraus ergibt sich auch $\mathfrak{R}^{M}(u)=\mathbf{0}$ und zugleich die Behauptung.

Die Sätze 1 und 2 gestatten die Folgerung:

Satz 3. Eine in $\bar{Z}$ eigentlich semidefinite Aufgabe ist genau dann eigentlich definit, wenn $\lambda=0$ kein Eigenwert ist.

Bemerkung. Satz 1 gilt auch in $Z$, da eine in $Z$ eigentlich definite Aufgabe eigentlich definit in $\bar{Z}$ ist 
Das Vorgehen bei Dück [3] gestattet den Beweis folgenden Satzes, auf dessen Begründung wir hier jedoch verzichten.

Satz 4. Ist bei einem in $Z$ eigentlich semidefiniten Problem für eine zulässige F'unktion $u(x)$ Gleichung (20) gültig, läßt sich $u(x)$ sogar $2 m$-mal stetig differenzieren, ist also eine streng zulässige Funktion.

Folgerung. Ein in $Z$ eigentlich semidefinites Problem, das nicht eigentlich definit in $Z$ ist, kann auch nicht eigentlich definit in $\bar{Z}$ sein. Damit gelten die Sätze 2 und 3 auch in $Z$.

5.2. Realităt der Eigenwerte. Die Realität der Eigenwerte wird durch den folgenden Satz gesichert.

SaTZ 5. Ein in $\bar{Z}$ eigentlich semidefinites Problem besitzt nur reelle Eigenwerte und die Eigenfunktionen können im reellen Raum $\bar{Z}$ gesucht werden.

Dieser Satz gilt natürlich auch wieder in $Z$.

Beweis. Wir nehmen an, daß $\lambda=s+i t$ Eigenwert und

$$
y(x)=u(x)+i v(x)
$$

eine zugehörige Eigenfunktion ist. Dabei sollen $s, t$ und $u(x), v(x)$ reelle Zahlen bzw. reelle Funktionen bezeichnen. Da die wesentlichen Randbedingungen (4) linear, homogen sind, ist

$$
W_{j}(u+i v)=W_{j}(u)+i W_{j}(v), \quad j=1, \ldots, k .
$$

Die Koeffizienten in den Randbedingungen sind reell, so daß $u(x)$ und $v(x)$ den wesentlichen Randbedingungen genügen müssen. Da sie auch die notwendigen Differenzierbarkeitseigenschaften besitzen, sind sie Elemente des reellen Raumes $\bar{Z}$.

Weiterhin ist

$$
\begin{gathered}
M(u+i v)-\lambda N(u+i v)=0, \\
R_{j}^{M}(u+i v)=\lambda R_{j}^{N}(u+i v), \quad j=1, \ldots, 2 m-k,
\end{gathered}
$$

und da die Koeffizientenfunktionen in der Differentialgleichung und die Konstanten in den Randbedingungen reell sind,

$$
\begin{gathered}
M(u-i v)-\bar{\lambda} N(u-i v)=0, \quad \overline{\hat{\lambda}}=s-i t, \\
W_{j}(u-i v)=0, \quad j=1, \ldots, k, \\
R_{j}^{M I}(u-i v)=\bar{\lambda} R_{j}^{N}(u-i v), \quad j=1, \ldots, 2 m-k .
\end{gathered}
$$

Aus (21) ergibt sich

$$
\langle u-i v, u+i v\rangle-\lambda[u-i v, u+i v]=0,
$$


woraus mit (17), (22)

$$
\begin{gathered}
(u-i v, u+i v)_{M}-\lambda(u-i v, u+i v)_{N}+\Re^{M}(u+i v) \mathfrak{x}(u-i v)- \\
-\lambda \Re^{N}(u+i v) \mathfrak{x}(u-i v)=0, \\
(u-i v, u+i v)_{M}-\lambda(u-i v, u+i v)_{\boldsymbol{N}}=\mathbf{0}
\end{gathered}
$$

gefolgert werden kann. Ebenso führt (23) zu der Gleichung

$$
(u+i v, u-i v)_{M}-\bar{\lambda}(u+i v, u-i v)_{N}=0 .
$$

Man überzengt sich leicht, daß wegen $u, v \in \bar{Z}$ auch $u+i v$ und $u-i v$ in den natürlichen Produktbildungen vertauscht werden dürfen, so da $\beta$ die beiden letzten Gleichungen die Folgerung

$$
(\lambda-\bar{\lambda})(u-i v, u+i v)_{N}=0
$$

gestatten. Nehmen wir $t \neq 0$ an, ist wegen $\lambda-\bar{\lambda}=2 t i$.

$$
(u-i v, u+i v)_{N}=0,
$$

also

$$
(u, u)_{N}+(v, v)_{N}=0 .
$$

Gleichzeitig muß mit (24)

gelten, was zu

$$
(u-i v, u+i v)_{M}=0
$$

$$
(u, u)_{M}+(v, v)_{M}=0
$$

und wegen der vorausgesetzten eigentlichen Semidefinitheit zu

$$
(u, u)_{M}=(v, v)_{M}=0
$$

führt. (26) hat weiter zur Folge, daß $(u, u)_{N},(v, v)_{N}$ von Null verschieden sein müssen und ein festes Vorzeichen besitzen. Das widerspricht aber (25). Damit mïssen alle existierenden Eigenwerte reell sein.

Wegen $\bar{\lambda}=\lambda$ und (21), (23) $\mathrm{mu} \beta$

$$
M u-\lambda N u=0, \quad M v-\lambda N v=0
$$

und ebenfalls

$$
R_{j}^{M}(u)=\lambda R_{j}^{N}(u), \quad R_{j}^{M}(v)=\lambda R_{j}^{N}(v), \quad j=1, \ldots, 2 m-k
$$

sein. Damit sind die reellen Funktionen $u(x), v(x)$ bereits selbst Eigenfunktionen zum Eigenwert $\lambda$. Sämtliche Eigenfunktionen lassen sich also aus reellen Eigenfunktionen linear komponieren. Zur Angabe eines Repräsentantensystems von Eigenfunktionen, aus dem sich sämtliche Eigenfunktionen linear komponieren lassen, genügt es, sich auf reelle Eigenfunktionen zu beschränken. Das soll fortan geschehen. 
5.3. Darstellung der Eigenwerte. Ist $\lambda_{i}$ Eigenwert und $y_{i}(x)$ eine zugehörige Eigenfunktion, gelten die Gleichungen

$$
\begin{gathered}
M y_{i}-\lambda_{i} N y_{i}=0, \\
W_{j}\left(y_{i}\right)=0, \quad j=1, \ldots, k, \\
R_{j}^{\mathcal{M}}\left(y_{i}\right)=\lambda_{i} R_{j}^{N}\left(y_{i}\right), \quad j=1, \ldots, 2 m-k .
\end{gathered}
$$

Aus (27) folgt unter Berücksichtigung von (28) für beliebige $u \in Z$

$$
\begin{gathered}
\left\langle u, y_{i}\right\rangle-\lambda_{i}\left[u, y_{i}\right]=0, \\
\left(u, y_{i}\right)_{M}+\mathfrak{R}^{M}\left(y_{i}\right) x(u)-\lambda_{i}\left(u, y_{i}\right)_{N}-\lambda_{i} \Re^{N}\left(y_{i}\right) \mathfrak{x}(u)=0, \\
\left(u, y_{i}\right)_{M}-\lambda_{i}\left(u, y_{i}\right)_{N}=\mathbf{0}, \quad u \in Z .
\end{gathered}
$$

SATZ 6. Bei einem in $\bar{Z}$ eigentlich semidefiniten Eigenwertproblem ist für eine zum Eigenwert $\lambda_{i}$ gehörige Eigenfunktion $y_{\imath}(x)$

und es gilt die Darstellung

$$
\left(y_{i}, y_{i}\right)_{N} \neq 0
$$

$$
\lambda_{i}=\frac{\left(y_{i}, y_{i}\right)_{M}}{\left(y_{i}, y_{i}\right)_{N}}
$$

Beweis. Wäre $\left(y_{i}, y_{i}\right)_{N}=0$, müßte wegen (29) für $u=y_{i}$ auch $\left(y_{i}, y_{i}\right)_{M}=0$ sein, was der Definitheitsvoraussetzung widerspricht. Aus (29) folgt dann die Darstellung (31).

5.4. Erklärung eines Rayleighschen Quotienten. Für jede zulässige Funktion $u(x)$, für die $(u, u)_{N} \neq 0$ ist, erklären wir einen Rayleigschen Quotient durch die Gleichung

$$
R(u)=\frac{(u, u)_{M}}{(u, u)_{N}} .
$$

SaTz 7. Bei Aufgaben mit Kamkeschen Randbedingungen ist der durch (32) definierte Rayleighsche Quotient gleich dem Kamkeschen Quotient ( $\left.{ }^{4}\right)$.

Das folgt unmittelbar aus den Definitionsgleichungen der beiden Quotienten, wenn man beachtet, daß bei diesen Aufgaben die reduzierten Dirichletschen Restteile mit den natürlichen übereinstimmen. Ebenso ergibt sich sofort aus (31) und (32):

SATZ 8. Der Rayleighsche Quotient einer zulässigen Funktion, für die $(u, u)_{N} \neq 0$ gilt, ist positiv, wenn die Aufgabe definit in $Z$ ist. Definite natürliche Eigenwertprobleme können nur positive Eigenwerte besitzen.

(4) Zur Definition des Kamkeschen Quotienten sei auf die einschlägige Literatur, insbesondere auf Dück [3], verwiesen. 
5.5. Orthogonalith̆tsrelationen. Wir beweisen jetzt die für die gesamten Betrachtungen grundlegenden Orthogonalitätsrelationen. - SATZ 9. Bezeichnen $\lambda_{i}, \lambda_{j}$ zwei verschiedene Eigenwerte und $y_{i}(x)$, $y_{j}(x)$ zugehörige Eigenfunltionen, gelten bei in $\bar{Z}$ eigentlich semidefiniten Aufgaben die Orthogonalitätsgleichungen

$$
\left(y_{i}, y_{j}\right)_{M}=\left(y_{i}, y_{j}\right)_{N}=0, \quad i \neq j, \quad \lambda_{i} \neq \lambda_{j} .
$$

Beweis. Wegen (29) ist

$$
\begin{aligned}
& \left(y_{j}, y_{i}\right)_{M}-\lambda_{i}\left(y_{j}, y_{i}\right)_{N}=0, \\
& \left(y_{i}, y_{j}\right)_{M}-\lambda_{j}\left(y_{i}, y_{j}\right)_{N}=0 .
\end{aligned}
$$

Daraus ergibt sich unmittelbar

$$
\left(\lambda_{i}-\lambda_{j}\right)\left(y_{i}, y_{j}\right)_{N}=0
$$

und infolge unserer Annahme $\lambda_{i} \neq \lambda_{j}$ ist $\left(y_{i}, y_{j}\right)_{N}=0$ und damit anch $\left(y_{i}, y_{j}\right)_{M}=0$ erfüllt.

6. Vertellung der Eigenwerte. Bezeichnet $z_{1}(x, \lambda), \ldots, z_{z_{m}}(x, \lambda)$ ein Hauptsystem von Lösungen der Differentialgleichung (1), ist jeder Wert $\lambda$ genau dann Eigenwert, wenn er Nullstelle der Eigenwertdeterminate

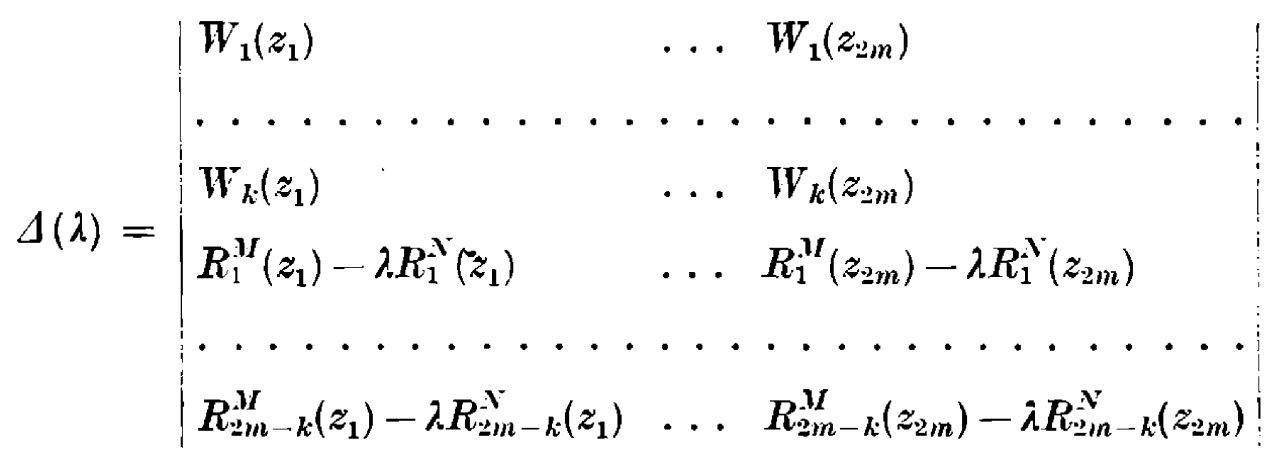

ist. Für die Vielfachheit $r$ eines Eigenwertes muß $1 \leqslant r \leqslant 2 m$ gelten.

SATZ 10. $\Delta(\lambda)$ ist eine ganze Funktion von $\lambda$.

Das ergibt sich unmittelbar wie in KIII. Dabei ist es belanglos. da $\beta$ die restlichen Randbedingungen von $\lambda$ linear abhängen.

SATZ 11. Bei einem in $\bar{Z}$ eigentlich semidefiniten natürlichen Eigenwertproblem ist die Vielfachheit eines Eigenwertes $\lambda_{i}$ gleich der Vielfachheit die $\lambda_{i}$ als Nullstelle von $\Lambda(\lambda)$ besitzt.

Beweis. Ist $\lambda_{i}$ ein Eigenwert der Vielfachheit $r$, hat $\Delta(\lambda)$ für $\lambda=\lambda_{i}$ den Rang $2 m-r$. Schreiben wir

$$
\begin{aligned}
U_{j}(u, \lambda) & =W_{j}(u), \quad j=1, \ldots, k, \\
U_{k+j}(u, \lambda) & =R_{j}^{M}(u)-\lambda R_{j}^{N}(u), \quad j=1, \ldots, 2 m-k,
\end{aligned}
$$


kann, wie KII zeigt, angenommen werden, daß die ersten $2 m-r$ Spalten von $\Delta(\lambda)$ für $\lambda=\lambda_{i}$ den Rang $2 m-r$ besitzen und das Hauptsystem von Lösungen der Differentialgleichung so gewählt ist, daß

$$
U_{\mu}\left(z_{j}\left(x, \lambda_{i}\right), \lambda_{i}\right)=0, \quad \mu=1, \ldots, 2 m, j=2 m-r+1, \ldots, 2 m
$$

gilt.

Differenzieren wir $\Delta(\lambda)$, so sind alle durch den Differentiationsprozeß entstehenden Determinanten für $\lambda=\lambda_{i}$ gleich Null, wenn nur eine der letzten $r$ Spalten unverändert auftritt. Daraus folgt $\Delta^{(j)}\left(\lambda_{i}\right)=0$ für $j<r$.

Wäre $\lambda_{l}$ eine $r$-fache Nullstelle von $\Delta(\lambda)$, müßte $\Delta^{(r)}\left(\lambda_{i}\right) \neq 0$ sein. Im Sinne eines indirekten Beweises nehmen wir

$$
S^{(r)}\left(\lambda_{i}\right)=0
$$

an. Beachten wir die obigen Bemerkungen über die Differentiation von $\Delta(\lambda)$, werden wir zu der Gleichung

$$
\Delta^{(r)}\left(\lambda_{i}\right)=\operatorname{det}\left(U_{\mu}\left(z_{1}, \lambda_{i}\right), \ldots, U_{\mu}\left(z_{2 m-r}, \lambda_{i}\right), \frac{d}{d \lambda} U_{\mu}\left(z_{2 m-r+1}, \lambda_{i}\right), \ldots, \frac{d}{d \lambda} U_{\mu}\left(z_{2 m}, \lambda_{i}\right)\right)
$$

geführt. Dann müssen wegen (3i) Zahlen $c_{i}$ existieren, die nicht sämtlich verschwinden und für die

$$
\sum_{j=1}^{2 m--r} c_{j} U_{\mu}\left(z_{j}, \lambda_{i}\right)+\sum_{j=2 m-r+1}^{2 m} c_{j} \frac{d}{d \lambda} U_{\mu}\left(z_{j}, \lambda_{i}\right)=0, \quad \mu=1, \ldots, 2 m
$$

ist. Da die ersten $2 m-r$ Spalten von $A^{(r)}\left(\lambda_{i}\right)$ den Rang $2 m-r$ haben, können sogar die Konstanten $c_{2 m-r+1}, \ldots, c_{2 m}$ nicht sämtlich verschwinden. Wir definieren drei Funktionen $s(x, \lambda), t(x, \lambda), z(x, \lambda)$ durch die Gleichungen

$$
\begin{gathered}
s(x, \lambda)=\sum_{j=2 m-r+1}^{2 m} c_{j} z_{j}(x, \lambda), \quad t(x, \lambda)=\frac{\partial s}{\partial \lambda}=\sum_{j=2 m-r+1}^{2 m} c_{j} \frac{\partial}{\partial \lambda} z_{j}(x, \lambda), \\
z(x, \lambda)=t(x, \lambda)+\sum_{j=1}^{2 m-r} c_{j} z_{j}(x, \lambda) .
\end{gathered}
$$

Als Funktionen des Hauptsystems sind $z_{2 m-r+1}\left(x, \lambda_{i}\right), \ldots, z_{2 m}\left(x, \lambda_{i}\right)$ wegen (36) sogar Eigenfunktionen zu $\lambda_{i}$. Also ist auch $s\left(x, \lambda_{i}\right)$ Eigenfunktion zum Eigenwert $\lambda_{i}$. Bezeichnet der Zeiger $\mu$ eine wesentliche Randbedingung, ist wegen (38)

$$
\left.\left.U_{\mu}\left(z\left(x, \lambda_{i}\right), \lambda_{i}\right)\right)=\sum_{j=1}^{2 m-r} c_{j} U_{\mu}\left(z_{j}\left(x, \lambda_{i}\right), \lambda_{i}\right)+\sum_{j=2 m-r+1}^{2 m} c_{j} \frac{d}{d \lambda} U_{\mu}\left(z_{j}\left(x, \lambda_{i}\right), \lambda_{i}\right)\right)=0 .
$$


Kennzeichnet $\mu$ eine restliche Randbedingung, ist der obige Schluß offenbar nicht gültig. Dann ist

$$
\begin{aligned}
& U_{\mu}\left(z\left(x, \lambda_{i}\right), \lambda_{i}\right) \\
& =\sum_{j=1}^{2 m-r} c_{j} U_{\mu}\left(z_{j}\left(x, \lambda_{i}\right), \lambda_{i}\right)+\sum_{j=2 m-r+1}^{2 m} c_{j} U_{\mu}\left(\left.\frac{\partial}{\partial \lambda} z_{j}(x, \lambda)\right|_{\lambda=\lambda_{i}}, \lambda_{i}\right) \\
& =\sum_{j=1}^{2 m-r} c_{j} U_{\mu}\left(z_{j}\left(x, \lambda_{i}\right), \lambda_{i}\right)+\sum_{j=2 m-r+1}^{2 m} c_{j}\left(R_{\mu}^{M}\left(\left.\frac{\partial z_{j}}{\partial \lambda}\right|_{\lambda=\lambda_{i}}\right)-\lambda_{i} R_{\mu}^{N}\left(\left.\frac{\partial z_{j}}{\partial \lambda}\right|_{\lambda=\lambda_{i}}\right)\right\} \\
& =\sum_{j=1}^{2 m=r} c_{j} U_{\mu}\left(z_{j}\left(x, \lambda_{i}\right), \lambda_{i}\right)+\sum_{j=2 m-r+1}^{2 m} c_{j} \frac{d}{d \lambda} U_{\mu}\left(z_{j}\left(x, \lambda_{i}\right), \lambda_{i}\right)+ \\
& =\sum_{j=2 m-r+1}^{2 m} c_{j} R_{\mu}^{N}\left(z_{j}\left(x, \lambda_{i}\right)\right)=R_{\mu}^{N}\left(\sum_{j=2 m-r+1}^{2 m} c_{j}^{2 m} z_{j}\left(x, \lambda_{i}\right)\right)=R_{\mu}^{N}\left(s\left(x, \lambda_{i}\right)\right) .
\end{aligned}
$$

Also muß bei Berücksichtigung von (3こ)

$$
R_{\mu}^{M}\left(z\left(x, \lambda_{i}\right)\right)-\lambda_{i} R_{\mu}^{N}\left(z\left(x, \lambda_{i}\right)\right)=R_{\mu}^{N}\left(s\left(x, \lambda_{i}\right)\right)
$$

gelten. $z\left(x, \lambda_{i}\right)$ gehört insbesondere auch zu $\bar{Z}$. Da $s\left(x, \lambda_{i}\right)$ Eigenfunktion $\mathrm{zu} \lambda_{i}$ ist, folgt aus (29)

$$
\left(z\left(x, \lambda_{i}\right), s\left(x, \lambda_{i}\right)\right)_{M}-\lambda_{i}\left(z\left(x, \lambda_{i}\right), s\left(x, \lambda_{i}\right)\right)_{N}=0 .
$$

Wie KII, beweist man die Gleichung

aus der sich

$$
M z(x, \lambda)-\lambda N z(x, \lambda)-N s(x, \lambda)=0,
$$

$$
\begin{aligned}
\left\langle s\left(x, \lambda_{i}\right), z\left(x, \lambda_{i}\right)\right\rangle- & \lambda_{i}\left[s\left(x, \lambda_{i}\right), z\left(x, \lambda_{i}\right)\right]-\left[s\left(x, \lambda_{i}\right), s\left(x, \lambda_{i}\right)\right]=0, \\
\left(s\left(x, \lambda_{i}\right), z\left(x, \lambda_{i}\right)\right)_{M}- & \lambda_{i}\left(s\left(x, \lambda_{i}\right), z\left(x, \lambda_{i}\right)\right)_{N}-\left[s\left(x, \lambda_{i}\right), s\left(x, \lambda_{i}\right)\right]+ \\
+ & +\left\{\mathfrak{R}^{M}\left(z\left(x, \lambda_{i}\right)\right)-\lambda_{i} \mathfrak{R}^{N}\left(z\left(x, \lambda_{i}\right)\right)\right\} \mathfrak{x}\left(s\left(x, \lambda_{i}\right)\right)=0
\end{aligned}
$$

ergibt. Das führt wegen (39), (40) zu

$$
\begin{gathered}
\mathfrak{R}^{N}\left(s\left(x, \lambda_{i}\right)\right) \mathfrak{x}\left(s\left(x, \lambda_{i}\right)\right)-\left[s\left(x, \lambda_{i}\right), s\left(x, \lambda_{i}\right)\right]=0, \\
\left(s\left(x, \lambda_{i}\right), s\left(x, \lambda_{i}\right)\right)_{N}=0 .
\end{gathered}
$$

Da $s\left(x, \lambda_{i}\right)$ Eigenfunktion ist, verstößt das gegen Satz 6 . 
Unmittelbar ergibt sich dann:

SATZ 12. Bei einem in $\bar{Z}$ eigentlich semidefiniten Problem ist $\Delta(\lambda) \not \equiv 0$.

SATZ 13. Eine in $\bar{Z}$ eigentlich semidefinite Aufgabe besitzt höchstens abzählbar viele Eigenwerte, die keinen Häufungspunkt im Endlichen haben.

SATZ 14. Bei einem in $\bar{Z}$ eigentlich semidefiniten Eigenwertproblem lassen sich die Eigenwerte in einer Folge

$$
\cdots \leqslant \lambda_{-2} \leqslant \lambda_{-1} \leqslant 0 \leqslant \lambda_{1} \leqslant \lambda_{2} \leqslant \cdots
$$

anordnen, wobei $\lambda_{i}$ die positiven Eigenwerte, $\lambda_{-i}$ die negativen Eigenwerte bezeichnen und jeder Eigenwert seiner Vielfachheit entsprechend oft angeführt wird. Ist $\lambda=0$ Eigenwert, wird er zum System der positiven Eigenwerte gezählt, wenn für seine zugehörigen Eigenfunktionen $y(x)$ die $U n$ gleichung $(y, y)_{N}>0$ gilt. Ist jedoch $(y, y)_{N}<0$, wird der Eigenwert Null zum System der negativen Eigenwerte gerechnet.

Bemerkung. Man beachte, da $\beta$ für alle zum Eigenwert $\lambda=0$ gehörige Eigenfunktionen $(y, y)_{N}$ ein festes Vorzeichen haben muß, da für diese Funktionen $(y, y)_{M}=0$ ist.

\section{Orthogonalsystem aus Eigenfunktionen.}

SaTZ 15. Zur Folge (41) gibt es bei in $\bar{Z}$ eigentlich semidefiniten Aufgaben ein System von Eigenfunktionen

$$
\ldots, y_{-2}(x), y_{-1}(x), y_{1}(x), y_{2}(x), \ldots,
$$

das der Orthogonalitätsrelation

$$
\left(y_{i}, y_{j}\right)_{N}=\left\{\begin{array}{rll}
0 & \text { für } & i \neq j, \\
\pm 1 & \text { für } & i=j,
\end{array} \quad i, j= \pm 1, \pm 2, \ldots\right.
$$

genügt. Dabei bezeichnet $y_{i}(x)$ eine zum Eigenwert $\lambda_{i}$ gehörige Eigenfunktion. Für $i=1,2, \ldots$ gilt in (42) die Normierung +1 und für $i=-1,-2, \ldots$ die Normierung -1 .

Beweis. Nach Satz 6 besteht offenbar für eine zum positiven Eigenwert $\lambda_{i}$ gehörige Eigenfunktion $y_{i}(x)$ die Ungleichung $\left(y_{i}, y_{i}\right)_{N}>0$ und $\left(y_{i}, y_{i}\right)_{N}<0$, wenn $y_{i}(x)$ zu einem negativen Eigenwert gehört, so daß die Normierung leicht möglich ist. Wegen Satz 9 gilt die Orthogonalitätsaussage bereits für zwei zu verschiedenen Eigenwerten gehörige Eigenfunktionen. Es bleibt nur zu zeigen, daß die zu demselben Eigenwert gehörigen Eigenfunktionen so orthogonalisiert werden können. Das ist aber möglich, weil das Schmidtsche Orthogonalisierungsverfahren wegen $\left(y_{i}, y_{i}\right)_{N} \neq 0$ durchgeführt werden kann.

SATZ 16. Ist das Eigenwertproblem eigentlich semidefinit in $\bar{Z}, m u \beta$ jedes System von Eigenfunktionen linear unabhängig sein, das entsprechend (42) orthonormiert ist und dessen Funktionen alle zu positiven oder negativen Eigenwerten gehören. 
Beweis. $y_{1}(x), \ldots, y_{j}(x)$ möge irgendein System von Eigenfunktionen bezeichnen, die alle zu positiven Eigenwerten gehören sollen. Wären diese Funktionen linear abhängig, würden nicht sämtlich verschwindende Konstanten $c_{1}, \ldots, c_{j}$ existieren, so daß

gilt. Dann müßte

$$
c_{1} y_{1}(x)+\ldots+c_{j} y_{j}(x)=0
$$

$$
\left(c_{1} y_{1}+\ldots+c_{j} y_{j}, c_{1} y_{1}+\ldots+c_{j} y_{j}\right)_{N}=0
$$

sein, was wegen (42) zu $c_{1}^{2}+\ldots+c_{j}^{2}=0$ führt und unserer Annahme widerspricht, daß nicht sämtliche $c_{i}$ verschwinden.

Entsprechend erfolgt der Beweis, wenn das System aus Eigenfunktionen besteht, die sämtlich zu negativen Eigenwerten gehören.

\section{Weitere Aussagen über natürliche Eigenwertprobleme.}

SATZ 17. Es gibt streng zulässige Funktionen $u(x)$, für die $(u, u)_{N} \neq 0$ ist, wenn das Eigenwertproblem eigentlich semidefinit in $\bar{Z}$ ist.

Beweis. Würde für jedes $u \in \bar{Z}(u, u)_{N}=0$ sein, müßte auch $(u+v$, $u+v)_{N}=0$ für jedes $v \epsilon \bar{Z}$, also $(v, u)_{N}=0$ gelten. Wählen wir $v(x)$ so, $\operatorname{da} \beta ¥(v)=0$ ist, folgt daraus $[v, u]=0$, womit wegen der Willkür von $v(x)$ leicht $N u=0$ gezeigt werden kann. Das führt aber wegen $g_{n}(x) \not \equiv 0$, wie bei KIII, zu einem Widerspruch.

SATZ 18. Bezeichnet $y_{1}(x), \ldots, y_{p}(x)$ ein System von Eigenfunktionen einer in $\bar{Z}$ eigentlich semidefiniten Aufgabe, das im Sinne von (42) orthogonalisiert ist, existieren streng zulässige Funktionen $u(x)$, die

$$
(u, u)_{N} \neq 0, \quad\left(u, y_{i}\right)_{N}=0 \quad \text { für } \quad i=1, \ldots, p
$$

genügen.

Beweis. Bezeichnet $u_{0}(x)$ eine beliebige streng zulässige Funktion und setzen wir

$$
u(x)=u_{0}(x)+\sum_{i=1}^{p} c_{i} y_{i}(x) \quad \text { mit } \quad c_{i}=-\frac{\left(u_{0}, y_{i}\right)_{N}}{\left(y_{i}, y_{i}\right)_{N}}
$$

erfüllt $u(x)$ die in (43) enthaltene Orthogonalitätsaussage. Wir müssen zeigen, daß durch passende Wahl von $u_{0}(x)$ auch $(u, u)_{N} \neq 0$ erreicht werden kann. Es ist leicht, die Gültigkeit der Gleichung

$$
(u, u)_{N}=\left(u_{0}, u_{0}\right)_{N}-\sum_{i=1}^{p} c_{i}^{2}\left(y_{i}, y_{i}\right)_{N}
$$

zu bestätigen. Würde also $(u, u)_{N}=0$ für jedes $u \epsilon \bar{Z}$ gelten, wäre

$$
\left(u_{0}, u_{0}\right)_{N}=\sum_{i=1}^{p} \frac{\left(u_{0}, y_{i}\right)_{N}^{2}}{\left(y_{i}, y_{i}\right)_{N}} \quad \text { für jedes } \quad u_{0} \epsilon \bar{Z} .
$$


Damit wäre auch

$$
\left(u_{0}+v, u_{0}+v\right)_{N}=\sum_{i=1}^{p} \frac{\left(u_{0}+v, y_{i}\right)_{N}^{2}}{\left(y_{i}, y_{i}\right)_{N}}
$$

und man folgert leicht

$$
\begin{gathered}
\left(u_{0}, v\right)_{N}=\sum_{i=1}^{p} \frac{\left(u_{0}, y_{i}\right)_{N}\left(v, y_{i}\right)_{N}}{\left(y_{i}, y_{i}\right)_{N}} \\
\left(v, u_{0}-\sum_{i=1}^{p} d_{i} y_{i}\right)_{N}=0 \quad \operatorname{mit} \quad d_{i}=\frac{\left(u_{0}, y_{i}\right)_{N}}{\left(y_{i}, y_{i}\right)_{N}}
\end{gathered}
$$

Wählen wir $v(x)$ aus $\bar{Z}$ beliebig, aber so, $\operatorname{da} \beta x(v)=0$ ist, kann

$$
\left[v, u_{0}-\sum_{i=1}^{p} d_{i} y_{i}\right]=0
$$

und wegen der Willkür von $v(x)$

$$
N\left(u_{0}-\sum_{i=1}^{p} d_{i} y_{i}\right) \doteq 0
$$

gezeigt werden. Daraus ergibt sich, wie in KIII, der Widerspruch.

\section{Eigenschaften der Greenschen Resolvente.}

\subsection{Existenz und Darstellung der Greenschen Resolvente.} Wir bezeichnen mit $G(x, \xi, \lambda)$ die Greensche Resolvente des Differentialausdrucks $M y-\lambda N y$ mit den Randbedingungen $W_{j}(y)=0, R_{j}^{M}(y)=\lambda R_{j}^{N}(y)$. $\lambda_{0}$ sei ein spezieller Wert des Parameters $\lambda$ und $G\left(x, \xi, \lambda_{0}\right)$ die Greensche Funktion von $M y-\lambda_{0} N y$ mit den Randbedingungen $W_{j}(y)=0, R_{j}^{M}(y)-$ $-\lambda_{0} R_{j}^{N}(y)=0$. Wegen (3) ist

$$
R_{j}^{M}(y)-\lambda_{0} R_{j}^{N}(y)=\sum_{i=0}^{2 m-1}\left\{\left(\alpha_{i j}^{M}-\lambda_{0} \alpha_{i j}^{N}\right) y^{(i)}(a)+\left(\beta_{i j}^{M}-\lambda_{0} \beta_{i j}^{N}\right) y^{i}(a)\right\}
$$

Wir haben also die Greensche Funktion eines linearen homogenen Differentialausdrucks mit Kamkeschen Randbedingungen zu bestimmen. Es gelten damit die bekannten Sätze. Insbesondere ist die Existenz der Greenschen Funktion $G\left(x, \xi, \lambda_{0}\right)$ gesichert, wenn die homogene Aufgabe

$$
\begin{gathered}
M y-\lambda_{0} N y=0, \\
W_{j}(y)=0, \quad j=1, \ldots, k, \\
R_{j}^{. M}(y)=\lambda_{0} R_{j}^{N}(y), \quad j=1, \ldots, 2 m-k
\end{gathered}
$$


keine eigentliche Lösung besitzt. Die Lösung der inlımogenen Aufgabe

$$
\begin{aligned}
& M y-\lambda_{0} N y=r(x), \\
W_{j}(y)= & \mathbf{0}, \quad j=1, \ldots, k, \\
R_{j}^{M I}(y)= & \lambda_{0} R_{j}^{N}(y), \quad j=1, \ldots, 2 m-k
\end{aligned}
$$

mit stetigem $r(x)$ stellt sich in der Form

$$
y(x)=\int_{a}^{b} G\left(x, \xi, \lambda_{0}\right) r(\xi) d \xi
$$

dar. Weiterhin muß für die Greensche Resolvente die Gleichung

$$
G(x, \xi, \lambda)=\gamma(x, \xi, \lambda)+\frac{Z(x, \xi, \lambda)}{\Delta(\lambda)}
$$

gelten. $A(\lambda)$ ist die durch (3t) gegebene Eigenwertdeterminante, die nach Satz 10 eine ganze Funktion in $\lambda$ ist. Für $\gamma(x, \xi, \lambda)$ und $Z(x, \xi, \lambda)$ gelten die in KII angegebenen Determinantendarstellungen; sie ergeben sich wieder als ganze Funktionen in $\lambda$. Damit $\operatorname{mu} \beta(x, \xi, \lambda)$ eine meromorphe Funktion von $\lambda$ sein, die Polstellen nur für solche Werte $\lambda$ besitzen kann, die Eigenwerte unseres Problems sind.

9.2. Symmetrie der Greenschen Resolvente. $Z u$ prüfen bleibt noch die Symmetrie der Greenschen Resolvente bezüglich $x$ und $\xi$

$$
G(x, \xi, \lambda)=G(\xi, x, \lambda) .
$$

Die Beweise in der Literatur benutzen die Selbstadjungiertheit der Aufgabe, die unter der Vorstellung des Vorliegens Kamkescher Randbedingungen definiert wird. Der bei Collatz [1] angegebene Beweis von Seifert läßt sich aber leicht auf die von uns untersuchten Probleme ausdehnen. Ist $\lambda_{0}$ nicht Eigenwert und bezeichnen $r(x), s(x)$ zwei beliebige stetige Funktionen, $u(x)$ und $v(x)$ die Lösungen der Randwertaufgaben

gilt

$$
\begin{array}{lll}
M u-\hat{\lambda}_{0} N u=r(x), & M v-\lambda_{0} N v=s(x), & \\
W_{j}(u)=0, & W_{j}(v)=0, & j=1, \ldots, k, \\
R_{j}^{M I}(u)=\lambda_{0} R_{j}^{N}(u), & R_{j}^{M}(v)=\lambda_{0} R_{j}^{N}(v), & j=1, \ldots, 2 m-k,
\end{array}
$$

$$
u(x)=\int_{a}^{b} G\left(x, \xi, \lambda_{0}\right) r(\xi) d \xi, \quad v(x)=\int_{a}^{b} G\left(x, \xi, \lambda_{0}\right) s(\xi) d \xi .
$$

$u(x), v(x)$ gehören zu $\bar{Z}$, wegen der Selbstadjungiertheit ist

$$
(u, v)_{M}-\lambda_{0}(u, v)_{N}-(v, u)_{M}+\lambda_{0}(v, u)_{N}=0 .
$$


Daraus ergibt sich

$$
\begin{aligned}
&\langle u, v\rangle-\lambda_{0}[u, v]-\langle v, u\rangle+\lambda_{0}[v, u]-\left\{\Re^{M}(v)-\lambda_{0} \Re^{N}(v)\right\} \mathfrak{x}(u)++\left\{\mathfrak{R}^{M I}(u)-\lambda_{0} \Re^{N}(u)\right\} \mathfrak{x}(v)=\mathbf{0}, \\
& \int_{a}^{b} u(x)\left\{1 L v-\lambda_{0} N v\right\} d x-\int_{a}^{b} v(x)\left\{M u-\lambda_{0} N u\right\} d x=\mathbf{0}, \\
& \int_{a}^{b} \int_{a}^{b} G\left(x, \xi, \lambda_{0}\right) r(\xi) s(x) d \xi d x-\int_{a}^{b} \int_{a}^{b} G\left(x, \xi, \lambda_{0}\right) s(\xi) r(x) d \xi d x=0, \\
& \int_{a}^{b} \int_{a}^{b}\left\{G\left(x, \xi, \lambda_{0}\right)-G\left(\xi, x, \lambda_{0}\right)\right\} r(\xi) s(x) d \xi d x=0 .
\end{aligned}
$$

Wegen der Willkür der Funktionen $r(x), s(x)$ kann die gesuchte Symmetrierelation sofort gefolgert werden.

\subsection{Ordnung der Polstellen der Greenschen Resolvente.} Wir wollen jetzt zeigen, daß bei einer in $\bar{Z}$ eigentlich semidefiniten Aufgabe die Greensche Resolvente höchstens Pole erster Ordnung besitzt. Ist $\lambda_{i}$ ein $r$-facher Eigenwert, so ist nach Satz $11 \lambda_{i}$ auch eine $r$-fache Nullstelle von $\Delta(\lambda)$. Wegen (44) kann damit $G(x, \xi, \lambda)$ an der Stelle $\lambda=\lambda_{i}$ höchstens eine Polstelle $r$-ter Ordnung besitzen. Da $\Delta(\lambda)$ eine ganze Funktion von $\lambda$ ist, liegen die Nullstellen von $\Delta(\lambda)$ isoliert. Für $a \leqslant x, \xi \leqslant b$ existiert eine Umgebung $0<\left|\lambda-\lambda_{i}\right| \leqslant \varrho$ von $\lambda_{i}$, in der die Greensche Resolvente die Entwicklung

$$
G(x, \xi, \lambda)=\sum_{j=-r}^{\infty} h_{j}(x, \xi)\left(\lambda-\lambda_{i}\right)^{j}
$$

gestattet. Bezeichnet $r(x)$ eine beliebige in $a \leqslant x \leqslant b$ stetige Funktion, besitzt die Randwertaufgabe

$$
\begin{gathered}
M y-\lambda N y=r(x), \\
W_{j}(y)=0, \quad j=1, \ldots, k, \\
R_{j}^{Y I}(y)=\lambda R_{j}^{-}(y), \quad j=1, \ldots, 2 m-k
\end{gathered}
$$

für jedes $\lambda$ aus einer gewissen Umgebung $0<\left|\lambda-\lambda_{i}\right| \leqslant \varrho_{1}$ eine Lösung $H(x, \lambda)$. Setzen wir

$$
H_{j}(x)=\int_{a}^{b} h_{j}(x, \xi) r(\xi) d \xi,
$$

folgt, wie in KII, daß $H(x, \lambda)$ die Entwicklung

$$
H(x, \lambda)=\sum_{j=-r}^{\infty} H_{j}(x)\left(\lambda-\lambda_{i}\right)^{j}
$$


gestattet, die gliedweise $2 m$-mal stetig nach $x$ differenziert werden darf, womit wir sie in die Differentialgleichung und die Randbedingungen eintragen dürfen:

$$
\begin{gathered}
\sum_{j=-r}^{\infty}\left(\lambda-\lambda_{i}\right)^{j}\left\{M H_{j}-\lambda N H_{j}\right\}=r(x), \\
\sum_{j=-r}^{\infty}\left(\lambda-\lambda_{i}\right)^{j} W_{\mu}\left(H_{j}\right)=0, \quad \mu=1, \ldots, k, \\
\sum_{j=-r}^{\infty}\left(\lambda-\lambda_{i}\right)^{j}\left\{R_{\mu}^{M}\left(H_{j}\right)-\lambda R_{\mu}^{N}\left(H_{j}\right)\right\}=0, \quad \mu=1, \ldots, 2 m-k .
\end{gathered}
$$

Ist $\varrho$ die kleinste Zahl, für die $H_{e}(x) \not \equiv 0$ ist, und nehmen wir $\varrho \leqslant-2$ an, liefert ein Koeffizientenvergleich, analog zu KII,

$$
\begin{aligned}
& M H_{Q}=\lambda_{i} N H_{e}, \quad M H_{\varrho+1}=\lambda_{i} N H_{Q+1}+N H_{\varrho}, \\
& W_{\mu}\left(H_{e}\right)=0, \quad W_{\mu}\left(H_{e+1}\right)=0, \quad \mu=1, \ldots, k, \\
& R_{\mu}^{M}\left(H_{\ell}\right)-\lambda_{i} R_{\mu}^{N}\left(H_{\ell}\right)=0, \quad R_{\mu}^{M}\left(H_{Q+1}\right)-\lambda_{i} R_{\mu}^{N}\left(H_{Q+1}\right)=R_{\mu}^{N}\left(H_{Q}\right), \\
& \mu=1, \ldots, 2 m-k .
\end{aligned}
$$

Weiter ist, wenn $\bar{H}_{\rho}(x)$ die zu $H_{e}(x)$ konjugiert komplexe Funktion bezeichnet,

$$
\begin{gathered}
M \bar{H}_{\varrho}=\lambda_{i} N \bar{H}_{\varrho}, \\
W_{\mu}\left(\bar{H}_{\varrho}\right)=0, \quad \mu=1, \ldots, k, \\
R_{\mu}^{M}\left(\bar{H}_{\varrho}\right)-\lambda_{i} R_{\mu}^{N}\left(\bar{H}_{\varrho}\right)=0, \quad \mu=1, \ldots, 2 m-k .
\end{gathered}
$$

Es ist leicht, die Gültigkeit der Gleichungen .

$$
\begin{aligned}
\left(\bar{H}_{\varrho}, H_{Q+1}\right)_{M} & =\left(H_{Q+1}, \bar{H}_{\varrho}\right)_{M}, \\
\left(\bar{H}_{\boldsymbol{Q}}, H_{\varrho+1}\right)_{N} & =\left(H_{Q+1}, \bar{H}_{\varrho}\right)_{N}
\end{aligned}
$$

zu bestätigen, und unter Berücksichtigung der erhaltenen Ergebnisse kann bei Verwendung der wiederholt benutzen Schlußweise gezeigt werden:

$$
\begin{aligned}
& 0=\left(\bar{H}_{e}, H_{e+1}\right)_{M}-\left(H_{e+1}, \bar{H}_{e}\right)_{M} \\
& =\left\langle\bar{H}_{e}, H_{Q+1}\right\rangle-\left\langle H_{Q+1}, \bar{H}_{e}\right\rangle-\mathfrak{R}^{M}\left(H_{e+1}\right) x\left(\bar{H}_{e}\right)+\mathfrak{R}^{M}\left(\bar{H}_{e}\right) \mathfrak{x}\left(H_{Q+1}\right) \\
& =\lambda_{i}\left[\bar{H}_{e}, H_{e+1}\right]+\left[\bar{H}_{e}, H_{e}\right]-\lambda_{i}\left[H_{e+1}, \bar{H}_{e}\right]-\mathfrak{R}^{M}\left(H_{e+1}\right) \mathfrak{x}\left(\bar{H}_{e}\right)+\mathfrak{R}^{M}\left(\bar{H}_{e}\right) \mathfrak{x}\left(H_{e+1}\right) \\
& =\lambda_{i}\left(\bar{H}_{e}, H_{\varrho+1}\right)_{N}+\left(\bar{H}_{e}, H_{e}\right)_{N}-\lambda_{i}\left(H_{e+1}, \bar{H}_{\varrho}\right)_{N}+\mathfrak{R}^{N}\left(H_{e}\right) \mathfrak{x}\left(\bar{H}_{e}\right)- \\
& -\left\{\mathfrak{R}^{M}\left(H_{\ell+1}\right)-\lambda_{i} \Re^{N}\left(H_{\varrho+1}\right)\right\} \mathfrak{x}\left(\bar{H}_{\varrho}\right)+\left\{\mathfrak{R}^{M}\left(\bar{H}_{\ell}\right)-\lambda_{i} \mathfrak{R}^{N}\left(\bar{H}_{\ell}\right)\right\} \mathfrak{x}\left(H_{\varrho+1}\right) \\
& =\left(\bar{H}_{e}, H_{e}\right)_{N}-\mathfrak{R}^{N}\left(H_{e}\right) \mathfrak{x}\left(\bar{H}_{e}\right)+\mathfrak{R}^{N}\left(H_{e}\right) \mathfrak{x}\left(\bar{H}_{\boldsymbol{e}}\right) \\
& =\left(\bar{H}_{Q}, H_{Q}\right)_{N} \text {. }
\end{aligned}
$$


$H_{e}(x)$ und $\bar{H}_{e}(x)$ sind offenbar Eigenfunktionen zu $\lambda_{i}$. Daher muß wegen $\left(\bar{H}_{\varrho}, H_{\varrho}\right)_{N}=0$ auch $\left(\bar{H}_{\varrho}, H_{e}\right)_{M}=0$ gelten. Schreiben wir

$$
H_{Q}(x)=u_{\mathrm{e}}(x)+i v_{\mathrm{Q}}(x),
$$

folgt daraus $\left(u_{e}, u_{e}\right)_{M}+\left(v_{e}, v_{e}\right)_{M}=0$ und mit der eigentlichen Semidefinitheit $\left(u_{e}, u_{e}\right)_{M}=\left(v_{e}, v_{e}\right)_{M}=0$. Ebenso finden wir aus $\left(\bar{H}_{\mathfrak{e}}, H_{\mathfrak{e}}\right)_{N}=0$ die Gleichung $\left(u_{e}, u_{e}\right)_{N}+\left(v_{e}, v_{e}\right)_{N}=0$. Wegen $\left(u_{e}, u_{e}\right)_{M}=\left(v_{e}, v_{e}\right)_{M}=0$, müssen $\left(u_{e}, u_{e}\right)_{N},\left(v_{e}, v_{e}\right)_{N}$ von Null verschieden sein und dasselbe Vorzeichen besitzen. Das ist ein Widerspruch. Also ist unsere Annahme $\varrho \leqslant-2$ nicht aufrechtzuerhalten und infolge der Willkür von $r(x)$ kann mit (45)

$$
h_{-r}(x, \xi)=\ldots=h_{-2}(x, \xi)=0
$$

gefolgert werden. Für die Greensche Resolvente gilt damit in einer gewissen Umgebung der Eigenwertstelle $\lambda_{i}$ die Entwicklung

$$
G(x, \xi, \lambda)=\frac{h_{-1}(x, \xi)}{\lambda-\lambda_{i}}+\sum_{j=0}^{\infty} h_{j}(x, \xi)\left(\lambda-\lambda_{i}\right)^{j} .
$$

9.4. Untersuchung des Residuums. Es soll jetzt noch das Residuum $h_{-1}(x, \xi)$ der Greenschen Resolvente untersucht werden. Das dazu in K II beschrittene Vorgehen ist zwangsläufig auch bei den von uns untersuchten Aufgaben gültig, weil dort die spezielle Gestalt der restlichen Randbedingungen nicht benutzt wird. Bezeichnet $y_{1}(x), \ldots, y_{r}(x)$ ein zum Eigenwert $\lambda_{i}$ gehöriges Repräsentantensystem von Eigenfunktionen, gilt für das Residuum der Greenschen Resolvente einer in $\bar{Z}$ eigentlich semidefiniten Aufgabe

$$
h_{-1}(x, \xi)=\sum_{i=1}^{r} \sum_{j=1}^{r} c_{i j} y_{i}(x) y_{j}(\xi), \quad a \leqslant x, \xi \leqslant b .
$$

Die $c_{i j}$ bezeichnen Konstanten, deren genauere Bestimmung sich für das Folgende erübrigt.

Die Greensche Resolvente gestattet damit wegen (46) in einer gewissen Uimgebung der Eigenwertstelle $\lambda_{i}$ die Entwicklung

$$
G(x, \xi, \lambda)=\sum_{i=1}^{r} \sum_{j=1}^{r} \frac{c_{i j} y_{i}(x) y_{j}(\xi)}{\lambda-\lambda_{i}}+G_{0}(x, \xi, \lambda) .
$$

$G_{0}(x, \xi, \lambda)$ ist eine meromorphe Funktion in $\lambda$, die aber an der Stelle $\lambda=\lambda_{i}$ regulär ist; insbesondere besitzt sie auch die in K II angebenen Differenzierbarkeitseigenschaften. 


\section{Rayleighsches Prinzip far den ersten positiven und negativen Eigenwert.}

SATz 19. Das Eigenwertproblem sei eigentlich definit in $\bar{Z}$. Gibt es streng zulässige Funktionen $u(x)$, für die

$$
(u, u)_{N}>0
$$

ist, existiert das Minimum des Rayleighschen Quotienten $R(u)$, wenn zu' Konkurrenz alle Funktionen aus $\bar{Z}$ zugelassen werden, die der Ungleichung (48) genügen. Dieser Minimalwert ist der kleinste positive Eigenwert:

$$
\lambda_{1}=\operatorname{Min}_{\substack{u \in \bar{Z} \\(u, u)_{N}>0}} R(u) .
$$

Jede zu $\lambda_{1}$ gehörige Eigenfunktion ist Lösung der Variationsaufgabe.

Existieren streng zulässige Funktionen, für die

$$
(u, u)_{N}<0
$$

gilt, besitzt $R(u)$ ein Maximum für alle Funktionen aus $\bar{Z}$, die (ธ̃0) genügen. Es ist

$$
\lambda_{1}=\operatorname{Max}_{\substack{u \in \bar{Z} \\(u, u)_{N}<0}} R(u) .
$$

Jede zum größten negativen Eigenwert $\lambda_{-1}$ gehörige Eigenfunktion ist Lösung dieser Variationsaufgabe.

Es existiert zumindest $\lambda_{1}$ oder $\lambda_{-1}$.

Ist das Eigenwertproblem eigentlich semidefinit, aber nicht eigentlich definit in $\bar{Z}$, ist $\lambda=0$ Eigenwert. Dieser Eigenwert wird durch (49) geliefert, wenn für die $u \in \bar{Z}$ mit $(u, u)_{M}=0$ die Ungleichung (48) gilt. Ist jedoch für diese $u(x)$ (50) erfüllt, wird der Eigenwert $\lambda=0$ durch (51) dargestellt.

Beweis. Bezeichnet $u(x)$ eine beliebige streng zulässige Funktion, die wir uns im folgenden fixiert denken und die (48) genügt - vorausgesetzt, daß solche Funktionen existieren -, ist wegen der eigentlichen Definitheit der Aufgabe $R=R(u)$ eine feste positive Zahl. Dann ist auch

$$
r(x)=M u-R N u
$$

eine feste in $a \leqslant x \leqslant b$ stetige Funktion und es sind

$$
\gamma_{j}=R_{i}^{M}(u)-R R_{j}^{N}(u), \quad j=1, \ldots, 2 m-k
$$

feste Zahlen. Gilt $r(x)=0$ und sind alle $\gamma_{j}=0$, ist $u(x)$ Eigenfunktion zum positiven Eigenwert $R$ und die Behauptung $R \geqslant \lambda_{1}$ bestimmt richtig.

Wir können also annehmen, daß nicht gleichzeitig

$$
r(x) \equiv 0 \quad \text { und } \quad \gamma_{j}=0 \quad \text { für } \quad j=1, \ldots, 2 m-k
$$


gilt und $R$ kein Eigenwert ist. Also hat die inhomogene Aufgabe

$$
\left\{\begin{array}{c}
M v-\lambda N v=r(x), \\
W_{j}(v)=0, \quad j=1, \ldots, k, \\
R_{j}^{M}(v)-\lambda R_{j}^{N}(v)=\gamma_{j}, \quad j=1, \ldots, 2 m-k
\end{array}\right.
$$

für jedes $\lambda$, das kein Eigenwert ist, genau eine Lösung $v=v(x, \lambda)$, die zu $\bar{Z}$ gehört und im Grundintervall nicht identisch verschwindet. Bezeichnet $v_{0}(x, \lambda)$ eine beliebige Funktion aus $\bar{Z}$, für die

$$
R_{j}^{\lambda I}\left(v_{0}\right)-\lambda R_{j}^{N}\left(v_{0}\right)=\gamma_{j}, \quad j=1, \ldots, 2 m-k
$$

gilt, und setzen wir

$$
w(x, \lambda)=v(x, \lambda)-v_{0}(x, \lambda),
$$

ist $w(x, \lambda)$ Lösung der Randwertaufgabe

$$
\begin{gathered}
M w-\lambda N w=s(x, \lambda) \quad \text { mit } \quad s(x, \lambda)=r(x)-M v_{0}-\lambda N v_{0}, \\
W_{\jmath}(w)=0, \quad j=1, \ldots, k, \\
R_{j}^{M I}(w)-\lambda R_{j}^{N}(w)=0, \quad j=1, \ldots, 2 m-k .
\end{gathered}
$$

$s(x, \lambda)$ ist stetig in $x$ und $\lambda$. Für $w(x, \lambda)$ gilt die Darstellung

$$
w(x, \lambda)=\int_{a}^{b} G(x, \xi, \lambda) s(\xi, \lambda) d \xi .
$$

Daraus ergibt sich

$$
v(x, \lambda)=v_{0}(x, \lambda)+\int_{a}^{b} G(x, \xi, \lambda) s(\xi, \lambda) d \xi .
$$

$v_{0}(x, \lambda)$ war beliebig in $\bar{Z}$, nur mußte (5̆ $)$ erfüllt sein. Wir können $v_{0}(x, \lambda)$ auch als stetige Funktion in $\lambda$ wählen. Wegen der Stetigkeit der Greenschen Resolvente für alle $\lambda$, die nicht Eigenwerte sind, folgt aus (56) auch die Stetigkeit von $v(x, \lambda)$ mit Ausnahme höchstens der Eigenwertstellen. Definieren wir eine Hilfsfunktion

$$
h(\lambda)=(v(x, \lambda), v(x, \lambda))_{M}-\lambda(v(x, \lambda), v(x, \lambda))_{N},
$$

muß damit auch $h(\lambda)$ stetig sein, wenn $\lambda$ nicht Eigenwert ist. Aus (57) finden wir in bekannter Weise mit (17), (18)

$$
\begin{aligned}
h(\lambda)=\langle v(x, \lambda), v(x, \lambda)\rangle- & \lambda[ \\
& v(x, \lambda), v(x, \lambda)]- \\
& -\left\{\Re^{M}(v(x, \lambda))-\lambda \Re^{N}(v(x, \lambda))\right\} \mathfrak{x}(v(x, \lambda)) .
\end{aligned}
$$


Erklären wir einen Spaltenvektor $\gamma$, dessen Komponenten die $\gamma_{1}, \ldots, \gamma_{2 m-k}$ aus (53) sind, ergibt sich wegen (54)

$$
\begin{aligned}
h(\lambda) & =\int_{a}^{b} v(x, \lambda)\{M v(x, \lambda)-\lambda N v(x, \lambda)\} d x-\gamma^{\prime} x(v(x, \lambda)), \\
& =\int_{a}^{b} v(x, \lambda) r(x) d x-\gamma^{\prime} x(v(x, \lambda)) .
\end{aligned}
$$

Bezeichnen $\lambda, \mu$ zwei Parameterwerte, die nicht Eigenwerte sind, ist unter Verwendung der wiederholt benutzten Schlußweise

$$
\begin{gathered}
h(\mu)-h(\lambda)=\int_{a}^{b}\{v(x, \mu)-v(x, \lambda)\} r(x) d x-\gamma^{\prime}\{x(v(x, \mu))-x(v(x, \lambda))\}, \\
=\int_{a}^{b} v(x, \mu)\{M v(x, \lambda)-\lambda N v(x, \lambda)\} d x- \\
-\int_{a}^{b} v(x, \lambda)\{M v(x, \mu)-\mu N v(x, \mu)\} d x-\gamma^{\prime}\{x(v(x, \mu))-x(v(x, \lambda))\} \\
=\langle v(x, \mu), v(x, \lambda)\rangle-\lambda\lceil v(x, \mu), v(x, \lambda)]-\langle v(x, \lambda), v(x, \mu)\rangle- \\
\quad-\mu\lceil v(x, \lambda), v(x, \mu)]-\gamma^{\prime}\{x(v(x, \mu))-x(v(x, \lambda))\} \\
=(v(x, \mu), v(x, \lambda)\rangle_{M}-\lambda\left(v(x, \mu),\left.v(x, \lambda)\right|_{N}-\right. \\
\quad-(v(x, \lambda), v(x, \mu)\rangle_{M}-\mu(v(x, \lambda), v(x, \mu))_{N}+ \\
\quad+\left\{\Re^{M}(v(x, \lambda))-\lambda \Re^{N}((x, \lambda))\right\} x(v(x, \mu))- \\
\quad-\left\{\Re^{M N}(v(x, \mu)\}-\mu \Re^{N}(v(x, \mu))\right\} x(v(x, \lambda))- \\
\quad-\gamma^{\prime}\{x(v(x, \mu))-x(v(x, \lambda))\} \\
=(\mu-\lambda)(v(x, \lambda), v(x, \mu)\rangle_{N} .
\end{gathered}
$$

Daraus folgt

$$
\begin{gathered}
\lim _{\mu \rightarrow \lambda} \frac{h(\mu)-h(\lambda)}{\mu-\lambda}=\lim _{\mu \rightarrow \lambda}(v(x, \lambda), v(x, \mu))_{N}, \\
h^{\prime}(\lambda)=(v(x, \lambda), v(x, \lambda))_{N} .
\end{gathered}
$$

Weiter ist

$$
\begin{aligned}
v(x, R) & =u(x), \\
h(R) & =(u, u)_{M}-R(u, u)_{N}=0, \\
h^{\prime}(R) & =(u, u)_{N}>0, \\
h(0) & =(v(x, 0), v(x, 0))_{M}>0 .
\end{aligned}
$$

Wie in K III, kann jetzt gezeigt werden, da $B$ im Intervall $0<\lambda \leqslant R$ ein Eigenwert liegen muß. Also ist $\lambda_{1} \leqslant R(u)$ für alle zugelassenen Funktionen $u(x)$ aus $\bar{Z}$ bewiesen. 
Die Existenz eines Minimums ergibt sich aus der Tatsache, daß wenn $y_{1}(x)$ eine zu $\lambda_{1}$ gehörige Eigenfunktion bezeichnet, $\lambda_{1}=R\left(y_{1}\right)$ ist.

Gibt es streng zulässige Funktionen $u(x)$ mit $(u, u)_{N}<0$, führen wir das Problem auf das eben behandelte zurück, indem wir die Aufgabe

betrachten.

$$
\begin{gathered}
M y-(-\lambda)(-N) y=0, \\
W_{j}(y)=0, \quad j=1, \ldots, k, \\
R_{j}^{M}(y)=(-\lambda)\left(-R_{j}^{N}(y)\right), \quad j=1, \ldots, 2 m-k
\end{gathered}
$$

Nach Satz 17 gibt es mindestens eine streng zulässige Funktion, für die entweder $(u, u)_{N}>0$ oder $(u, u)_{N}<0$ gilt. Damit ist die Existenz wenigstens eines Eigenwertes $\lambda_{1}$ oder $\lambda_{-1}$ bewiesen.

Ist das Eigenwertproblem eigentlich semidefinit in $\bar{Z}$, aber nicht eigentlich definit, muß nach Satz $3 \lambda=0$ Eigenwert sein. Es ist klar, daß dieser Eigenwert durch (49) oder (51) geliefert wird, je nachdem für die $u \in \bar{Z}$ mit $(u, u)_{M}=0$ (48) oder (50) gilt.

\section{Rayleighsches Prinzip für die höheren positiven und negativen Eigenwerte.}

Satz 20. Wir denken uns die positiven und negativen Eigenwerte $\lambda_{i}, \lambda_{-i}$ im Sinne von Satz 14 in einer Folge angeordnet und die zugehörigen Eigenfunktionen $y_{i}(x), y_{-i}(x)$ wie in Satz 15 orthonormiert.

Ist das Eigenwertproblem eigentlich definit in $\bar{Z}$, löst die Eigenfunktion $y_{p}(x)$ die Variationsaufgabe, den Rayleighschen Quotient $R(u)$ zu einem Minimum zu machen, wenn $u(x)$ den Bereich aller streng zulässigen Funktionen durchläuft, die der Orthogonalitätsrelation

$$
\left(u, y_{i}\right)_{N}=0, \quad i=1, \ldots, p-1
$$

und der Ungleichung (48) genügen. Der zugehörige Minimalwert ist der auf $\lambda_{p_{-1}}$ folgende nächste positive Eigenwert

$$
\lambda=\operatorname{Min}_{\substack{u \in \tilde{Z} \\(u, u)_{N}>0 \\\left(u, y_{i}\right)_{N}=0, i=1, \ldots, p-1}} R(u) .
$$

Ebenso löst $y_{-p}(x)$ die Maximumsaufgabe von $R(u)$, wenn es streng zulässige F'unktionen $u(x)$ gibt, welche die Orthogonalitätsgleichung

$$
\left(u, y_{-i}\right)_{N}=0, \quad i=1, \ldots, p-1
$$

und die Ungleichung (50) erfüllen. Dann ist

$$
\lambda_{-p}=\operatorname{Max}_{\substack{u \in \bar{Z} \\(u, u)_{N}<0 \\\left(u, y_{-i}\right)_{N}=0, i=1, \ldots, p-1}} R(u) .
$$


Mehrfache Eigenwerte werden im Sinne von Satz 14 stets entsprechend ihrer Vielfachheit gezählt.

Es existieren unendlich viele Eigenwerte.

Ist das Eigenwertproblem eigentlich semidefinit, aber nicht eigentlich definit in $\bar{Z}$ und $r_{1}$ die Vielfachheit des Eigenwertes Null, gilt folgendes:

1) Erfüllen alle $u \in \bar{Z}$ mit $(u, u)_{M}=0$ die Ungleichung (48), gilt das Variationsprinzip für $\lambda_{p}$ unverändert wie oben, während für $\lambda_{-p}$ (einschließlich $p=1$ ) die Variationsaufgabe nicht mit der Nebenbedingung (61), sondern mit der veränderten

$$
\left(u, y_{-i}\right)_{N}=0, \quad i=-r_{1}, \ldots,-1,1, \ldots, p-1
$$

$z u$ begleiten ist.

2) Erfüllen alle $u \in \bar{Z}$ mit $(u, u)_{M}=0$ (50), besteht das Variationsprinzip für $\lambda_{-p}$ unverändert, jedoch ist die Variationsaufgabe für $\lambda_{p}$ mit der Nebenbedingung

$$
\left(u, y_{i}\right)_{N}=0, \quad i=-r_{1}, \ldots,-1,1, \ldots, p-1,
$$

und nicht mit (59), zu betrachten.

B ew eis. Es wird zunächst vorausgesetzt, daß das Eigenwertproblem eigentlich definit ist und es werden die positiven Eigenwerte behandelt.

I. Gibt es streng zulässige Funktionen, die (48) genügen, existiert nach Satz 19 ein kleinster positiver Eigenwert $\lambda_{1}$, der durch (49) geliefert wird.

II. Ist $\lambda_{1}$ ein $r_{1}$-facher Eigenwert, ist die Behauptung für jedes $p$ aus $1<p \leqslant r_{1}$ richtig. Das folgt wie in K III.

III. Nach Satz 18 gibt es streng zulässige Funktionen, für die (59) mit $p=r_{1}+1$ und $(u, u)_{N} \neq 0$ gilt. Existieren unter diesen Funktionen solche mit $(u, u)_{N}>0$ - das ist bestimmt der Fall, wenn es weitere positive Eigenwerte gibt -, ist der Bereich der zur Konkurrenz zugelassenen Funktionen nicht leer. Wir wollen zeigen, daß dann ein weiterer positiver Eigenwert existiert. Dazu untersuchen wir den Fall $p=r_{1}+1$, wobei auch $r_{1}=1$ sein darf.

1. Nach Satz 19 ist $\lambda_{r_{1}} \leqslant R(u)$ für jede streng zulässige Funktion. Wir wollen zeigen, daß sogar $\lambda_{r_{1}}<R(u)$ gelten muß, wenn $u(x)$ noch der Orthogonalitätsgleichung (59) genügt. Wegen (59) kann $u(x)$ offenbar nicht Eigenfunktion zum Eigenwert $\lambda_{r_{1}}$ sein. $u(x)$ muß also einer inhomogenen Aufgabe

$$
\begin{gathered}
M u-\lambda_{r_{1}} N u=r(x), \\
W_{\mathcal{g}}(u)=0, \quad j=1, \ldots, k, \\
R_{j}^{M}(u)-\lambda_{r_{1}} R_{j}^{N}(u)=\gamma_{j}, \quad j=1, \ldots, 2 m-k
\end{gathered}
$$


genügen, und es darf nicht gleichzeitig $r(x):=0$ und $\gamma_{j}=0$ für alle $j$ gelten. Dann gibt es streng zulässige Funktionen $v(x)$, für die

$$
(u, v)_{M}-\lambda_{r_{1}}(u, v)_{N} \neq 0, \quad\left(v, y_{i}\right)_{N}=0, \quad i=1, \ldots, p-1
$$

ist. Denn bezeichnet $v_{0}(x)$ eine beliebige streng zulässige Funktion und setzen wir

$$
v(x)=v_{0}(x)+\sum_{i=1}^{p-1} c_{i} y_{i}(x) \quad \text { mit } \quad c_{i}=-\frac{\left(v_{0}, y_{i}\right)_{N}}{\left(y_{i}, y_{i}\right)_{N}}
$$

ist die in (65) enthaltene Orthogonalitätsaussage erfüllt.

Wenn $r(x)$ in (63) nicht identisch verschwindet, existiert im Grundintervall ein Intervall $\alpha<x<\beta$, in dem

$$
M u-\lambda_{r_{1}} N u \neq 0
$$

ist und dort ein festes Vorzeichen hat. Wählen wir $v_{0}(x) 2 m$-mal in $a \leqslant x \leqslant b$ stetig differenzierbar, $>0$ in $a<x<\beta,=0$ außerhalb dieses Intervalles und $\mathfrak{x}\left(v_{0}\right)=0$, gilt

$$
\begin{gathered}
\left(u, v_{0}\right)_{M}-\lambda_{r_{1}}\left(u, v_{0}\right)_{N}=\int_{a}^{\beta} v_{0}(x)\left\{M u-\lambda_{r_{1}} N u\right\} d x \neq 0, \\
(u, v)_{M}-\lambda_{r_{1}}(u, v)_{N}=\left(u, v_{0}\right)_{M}-\lambda_{r_{1}}\left(u, x_{0}\right)_{N}+c, \\
c=\sum_{i=1}^{p-1} c_{i}\left\{\left(u, y_{i}\right)_{M}-\lambda_{r_{1}}\left(u, y_{i}\right)_{N}\right\} .
\end{gathered}
$$

Wegen der Orthogonalitätsgleichung (59) ist $c=0$ und damit auch die erste Aussage von (6כ) bewiesen.

Ist $r(x)$ in (63) identisch Null, dürfen nicht alle $\gamma_{j}$ aus (64) verschwinden. Es ist dann

$$
\left(u, v_{0}\right)_{M I}-\lambda_{r_{1}}\left(u, v_{0}\right)_{N}=-\left\{\mathfrak{R}^{M I}(u)-\lambda_{r_{1}} \mathfrak{R}^{N}(u)\right\} \mathfrak{x}\left(v_{0}\right) .
$$

Wählen wir $v_{0}(x)$ in $\bar{Z}$ beliebig, aber $\boldsymbol{x}\left(v_{0}\right) \neq \mathbf{0}$, ist

$$
\left(u, v_{0}\right)_{M}-\lambda_{r_{1}}\left(u, v_{0}\right)_{x} \neq 0 .
$$

Wieder gilt (66), (67) und $c=0$, so daß auch in diesem Falle die Behauptung richtig ist.

Mit $u(x)$ und $v(x)$ genügt auch $u(x)+\varepsilon v(x)$ der Orthogonalitätsgleichung, und es ist sogar für hinreichend kleine $|\varepsilon|$ die Ungleichung $(u+\varepsilon v, u+\varepsilon v)_{N}>0$ gültig. Daher ist nach Satz $19 \lambda_{r_{1}} \leqslant R(u+\varepsilon v)$, d. h.

$$
(u+\varepsilon v, u+\varepsilon v)_{M}-\lambda_{r_{1}}(u+\varepsilon v, u+\varepsilon v)_{x} \geqslant 0 .
$$

Aus $R(u)=\lambda_{r_{1}}$ würde

$$
2 \varepsilon\left\{(u, v)_{M}-\lambda_{r_{L}}(u, v)_{\Lambda}\right\} \geqslant-\varepsilon^{2}\left\{(v, v)_{M}-\lambda_{r_{1}}(v, v)_{\Lambda}\right\}
$$


folgen, was zu (65) im Widerspruch steht. Die Annahme $\lambda_{r_{1}}=R(u)$ für eine streng zulässige Funktion, die der Orthogonalitätsgleichung ( 59$)$ genügt, kann nicht aufrechterhalten werden.

2. Analog zum Beweis von Satz 19 soll nun gezeigt werden, daß im Interval $\lambda_{r_{1}}<\lambda \leqslant R$ mindestens ein weiterer Eigenwert liegt. Dazu definieren wir eine Funktion $r(x)$ und Zahlen $\gamma_{1}, \ldots, \gamma_{2 m-k}$ durch (52), (53). Wir dürfen wieder annehmen, daß nicht gleichzeitig $r(x) \equiv 0$ und $\gamma_{1}=\ldots=\gamma_{2 m-k}=0$ gilt und $R$ kein Eigenwert ist. Durch (54) erklären wir eine Funktion $v(x, \lambda)$ und durch $(57)$ die Hilfsfunktion $h(\lambda)$. Dann ist wieder:

$$
\begin{aligned}
& h^{\prime}(\lambda)=(v(x, \lambda), v(x, \lambda))_{N} \text { für alle } \lambda, \text { die nicht Eigenwerte sind, } \\
& h(R)=0, \\
& h(\lambda)<0, h^{\prime}(\lambda)>0,(v(x, \lambda), v(x, \lambda))_{N}>0 \text { für alle } \lambda \text { aus } R_{0}<\lambda \leqslant R,
\end{aligned}
$$
wenn dieses Intervall frei von Eigenwerten ist.

Weiterhin gilt für $i=1, \ldots, p-1$

$$
\begin{aligned}
\lambda\left(v, y_{i}\right)_{N} & =\lambda\left[y_{i}, v\right]-\lambda \Re^{N}(v) \mathfrak{x}\left(y_{i}\right) \\
& =\left\langle y_{i}, v\right\rangle-\int_{a}^{b} y_{i}(x) r(x) d x-\lambda \Re^{N}(v) \mathfrak{x}\left(y_{i}\right) \\
& =\left\langle y_{i}, v\right\rangle-\left\langle y_{i}, u\right\rangle+R\left[y_{i}, u\right]-\lambda \Re^{N}(v) \mathfrak{x}\left(y_{i}\right) \\
& =\left(y_{i}, v\right)_{M}-\left(y_{i}, u\right)_{M}+R\left(y_{i}, u\right)_{N}+\left\{\mathfrak{R}^{M}(v)-\lambda \Re^{N}(v)\right\} \mathfrak{x}\left(y_{i}\right)- \\
& =\lambda_{i}\left(v, y_{i}\right)_{N^{N}}-\left(y_{i}, u\right)_{M}+R\left(y_{i}, u\right)_{N} .
\end{aligned}
$$

Die Orthogonalitätsrelation (59) und $\lambda \neq \lambda_{\imath}$ führen uns zu

$$
\left(v, y_{i}\right)_{N}=0, \quad i=1, \ldots, p-1 .
$$

Mit $u(x)$ genügt also auch $v(x, \lambda)$ für jedes $\lambda$ aus $\lambda_{r_{1}}<\lambda \leqslant R$ der Orthogonalitätsgleichung.

3. Beim Beweis von Satz 19 war unmittelbar klar, daß $h(0)>0$ ist, da Null kein Eigenwert ist. Jetzt ist der linke Endpunkt des Intervalles $\lambda_{r_{1}}<\lambda \leqslant R$ Eigenwert, was zusätzliche Untersuchungen notwendig macht. Es existiert aber in einer gewissen Umgebung des Eigenwertes $\lambda_{r_{1}}$ die Greensche Resolvente und $v(x, \lambda)$. Wegen $(47)$ und (56) gilt in dieser Umgebung

$$
\begin{aligned}
& v(x, \lambda)=v_{0}(x, \lambda)+ \\
& \quad+\int_{a}^{b} G_{0}(x, \xi, \lambda) s(\xi, \lambda) d \xi+\sum_{i=1}^{r_{1}} \sum_{j=1}^{r_{1}} \frac{c_{i j}}{\lambda-\lambda_{r_{1}}} y_{i}(x) \int_{a}^{b} y_{j}(\xi) s(\xi, \lambda) d \xi .
\end{aligned}
$$


$v_{0}(x, \lambda)$ mußte beim Beweis von Satz 19 eine stetige Funktion in $\lambda$ sein, die der Gleichung (55) genügt. Wir können $v_{0}(x, \lambda)$ offenbar auch so wählen, daß

$$
\left(v_{0}, y_{i}\right)_{N}=0, \quad i=1, \ldots, p-1
$$

ist. Aus (59) folgt

$$
\begin{aligned}
\int_{a}^{b} r(x) y_{i}(x) d x & =\left\langle y_{i}, u\right\rangle-R\left[y_{i}, u\right] \\
& =\left(y_{i}, u\right)_{M}-R\left(y_{i}, u\right)_{N}+\left\{\Re^{M}(u)-R \Re^{N}(u)\right\} \mathfrak{x}\left(y_{i}\right) \\
& =\left\{\mathfrak{R}^{M}(u)-R \Re^{N}(u)\right\} \mathfrak{x}\left(y_{i}\right) .
\end{aligned}
$$

Das führt zusammen mit (53), (55), (69) und der Definitionsgleichung von $s(x, \lambda) \mathrm{zu}$

$$
\begin{aligned}
& \int_{a}^{b} s(x, \lambda) y_{i}(x) d x=\int_{a}^{b}\left\{r(x)-M v_{0}(x, \lambda)+\lambda N v_{0}(x, \lambda)\right\} y_{i}(x) d x \\
& =\left\{\mathfrak{R}^{M}(u)-R \mathfrak{R}^{N}(u)\right\} \mathfrak{x}\left(y_{i}\right)-\left(y_{i}, v_{0}\right)_{M}+\lambda\left(y_{i}, v_{0}\right)_{N}- \\
& =0 \text {. } \\
& -\left\{\Re^{M K}\left(v_{0}\right)-\lambda \Re^{N}\left(v_{0}\right)\right\} \mp\left(y_{i}\right)
\end{aligned}
$$

Mit (68) erhalten wir

$$
v(x, \lambda)=v_{0}(x, \lambda)+\int_{a}^{b} G_{0}(x, \xi, \lambda) s(\xi, \lambda) d \xi
$$

in einer gewissen Umgebung von $\lambda_{r_{1}}$. Die Singularität an der Stelle $\lambda_{r_{1}}$ ist herausgefallen, da $G_{0}(x, \xi, \lambda)$ dort regulär ist. Der Rest des Beweises ergibt sich nun wie in $K$ III.

IV. In der bei $\mathrm{K}$ III geschilderten Weise kann fortgefahren werden, solange es Funktionen aus $Z$ gibt, für die $(u, u)_{N}>0$ und die Orthogonalitätsrelation erfüllt sind.

Der Beweis für die negativen Eigenwerte ergibt sich wie in Satz 19, indem das Problem auf das eben behandelte zurückgeführt wird.

Wie in K III folgt, daß dieses Verfahren höchstens nach einer Seite abbrechen kann, womit die Existenz von unendlich vielen Eigenwerten gezeigt ist. Damit ist der Satz für eigentlich definite Aufgaben vollständig bewiesen.

Ist das Problem eigentlich semidefinit, aber nicht eigentlich definit in $\bar{Z}$, kann wieder vollkommen, wie in $K$ III, geschlossen werden.

\section{Erweiterung des Bereiches der beim Rayleighschen Prinzip zur Konkurrenz zugelassenen Funktionen.}

SATZ 21. Ist das Eigenwertproblem eigentlich definit bzw. eigentlich semidefinit in $Z$, gelten die Sätze 19 und 20 sogar dann, wenn die zur Konkurrenz zugelassenen Funktionen aus $Z$ und nicht nur aus $\bar{Z}$ entnommen werden. 
Beweis. Von Dück wurde in [3] mittels eines Approximationssatzes nach einem Gedanken von Lehmann das Kamkesche Prinzip bewiesen, indem es auf das Variationsprinzip in K III zurückgeführt wurde. Dieser Gedanke führt auch hier zum Beweis des Satzes, wenn die Funktionen aus $Z$ durch geeignete Funktionen aus $\bar{Z}$ approximiert werden und dann in der bei Dück skizzierten Weise geschlossen wird. Auf eine Ausführung des Beweises soll verzichtet werden.

Folgerung bezüglich des Kamkeschen Prinzips: Liegen Aufgaben mit Kamkeschen Randbedingungen vor, ist nach Satz 7 der Kamkesche Quotient gleich dem Rayleighschen Quotient. Bei Dück [3] wurde das Kamkesche Prinzip für in $Z$ definite Aufgaben bewiesen. Jetzt ist dieses Prinzip auf in $Z$ eigentlich semidefinite Aufgaben ausgedehnt worden. Das bei Dück beschrittenè Vorgehen hätte sich leicht auf eigentlich semidefinite Aufgaben übertragen lassen, weil die dazu benötigen Sätze in V in K III bereitgestellt sind. Bei Dück [3] war aber der Vergleich mit der K-Definitheit das Hauptanliegen der Arbeit.

Bemerkung. Die Erweiterung des Bereiches der zur Konkurrenz zugelassenen Funktionen durch Satz 21 ist längst nicht von der Bedeutung, wie die Erweiterung des Prinzips in K III durch das Kamkesche Prinzip in [7]. Hier liegt nur eine Milderung der Differenzierbarkeitsvoraussetzungen vor, die praktisch weniger ins Gewicht fällt.

\section{Bemerkungen über das Vorzeichen der Eigenwerte.}

SATZ 22. Gibt es in $a \leqslant x \leqslant b$ ein Teilintervall $\alpha \leqslant x \leqslant \beta$, in dem.

$$
g_{i}(x) \geqslant 0, \quad i=0,1, \ldots, n
$$

und mindestens eine dieser Funktionen $>0$ ist, existieren unendlich riele positive Eigenicerte.

Gilt in (70) ïberall das entgegengesetzte Ungleichheitszeichen, existieren unendlich viele negative Eigenwerte.

Gibt es Teilintervalle von beiden Arten, existieren unendlich viele positive und negative Eigenwerte.

Beweis. Bezeichnet $u_{0}(x)$ eine beliebige streng zulässige Funktion und $y_{1}(x), \ldots, y_{p}(x)$ ein im Sinne von (42) orthogonalisiertes System von Eigenfunktionen, erfüllt

$$
u(x)=u_{0}(x)+\sum_{i=1}^{p} c_{i} y_{i}(x) \quad \text { mit } \quad c_{i}=-\frac{\left(u_{0}, y_{i}\right)_{N}}{\left(y_{i}, y_{i}\right)_{N}}
$$

die in (43) enthaltene Orthogonalitätsaussage. Beim Beweis von Satz 18 wurde gezeigt, daß durch Wahl von $u_{0}(x)(u, u)_{N} \neq 0$ erreicht werden kann. Jetzt muß bewiesen werden, daß $u_{0}(x)$ so wählbar ist, daß wegen (70) sogar $(u, u)_{N}>0$ jst, was, wie in K III, geschehen kann. 


\section{Festlegung der Eigenwerte nach Courant.}

Satz 23. Das Eigenwertproblem sei eigentlich definit in Z. Mit $w_{1}(x), \ldots, w_{p-1}(x)$ wird ein beliebiges System $2 n$-mal stetig differenzierbarer Funktionen bezeichnet, die am Rande auch die Bildung $\Re^{N}\left(w_{i}\right)$ gestatten. Wir bestimmen mit diesen Funktionen die untere Grenze

$$
m\left(w_{1}, \ldots, w_{p-1}\right)=\underline{\text { fin }} R(u)
$$

und die obere Grenze

$$
M\left(w_{1}, \ldots, w_{p-1}\right)=\overline{\text { fin }} R(u)
$$

des Rayleighschen Quotienten für alle Funktionen aus Z, die der Orthogonalitätsrelation

$$
\int_{a}^{b} u(x) N w_{i}(x) d x-\mathfrak{R}^{N}\left(w_{i}\right) \mathfrak{x}(u)=0, \quad i=1, \ldots, p-1
$$

genügen und für die im Falle (71)

und im Falle (72)

$$
(u, u)_{x}>0
$$

gilt. Es ist

$$
(u, u)_{N}<0
$$

$$
\begin{gathered}
m\left(w_{1}, \ldots, w_{p-1}\right) \leqslant \lambda_{p}, \\
M\left(w_{1}, \ldots, w_{p-1}\right) \geqslant \lambda_{-p},
\end{gathered}
$$

soweit diese Eigenwerte existieren.

Werden noch die Funktionen $w_{1}(x), \ldots, w_{p-1}(x)$ variiert, gilt.

$$
\begin{aligned}
& \lambda_{p}=\underset{w_{i}}{\operatorname{Max}} m\left(w_{1}, \ldots, w_{p-1}\right), \\
& \lambda_{-p}=\operatorname{Min}_{w_{i}} M\left(w_{1}, \ldots, w_{p-1}\right) .
\end{aligned}
$$

Ist das Eigenwertproblem eigentlich semidefinit in $Z$, aber nicht eigesatlich definit, ist $\lambda=0$ Eigenwert. Dann gilt folgendes:

1) Erfüllen alle $u \in Z$ mit $(u, u)_{M}=0$ die Ungleichung $(u, u)_{N}>0$, besteht das Variationsprinzip für die $\lambda_{p}$ unverändert, während wir zur F'estlegung von $\lambda_{-p}$ (einschließlich $p=1$ ) die Variationsaufgabe mit der veränderten Nebenbedingung

$$
\int_{a}^{b} u(x) N w_{i}(x) d x-\mathfrak{R}^{N}\left(w_{i}\right) \mathfrak{x}(u)=0, \quad i=-r_{1}, \ldots,-1,1, \ldots, p-1
$$

$z u$ betrachten haben. Dabei bezeichnet $r_{1}$ die Vielfachheit des Eigenwertes $\lambda=0$ und $w_{-r_{1}}(x), \ldots, w_{-1}(x), w_{1}(x), \ldots, w_{p-1}(x)$ ein System von $r_{1}+p-1$ Funktionen der obigen Art. 
2) Ist für alle $u \in Z$, für die $(u, u)_{M}=0$ gilt, $(u, u)_{N}<0$, besteht das Variationsprinzip für $\lambda_{-p}$ unverändert, während die Aufgabe für $\lambda_{p}$ jetzt mit der Nebenbedingung (78) zu begleiten ist.

Beweis. Zum Beweis von (74) geben wir eine zulässige Funktion $u(x)$ an, welche die geforderten Eigenschaften besitzt und für die $R(u) \leqslant \lambda_{p}$ ist. Dazu bilden wir

$$
u(x)=\sum_{i=1}^{p} c_{i} y_{i}(x)
$$

wobei $y_{i}(x)$ die im Sinne von Satz 15 orthonormierten Eigenfunktionen bezeichnen. Die $c_{i}$ werden so bestimmt, daß die Orthogonalitätsrelation (73) erfüllt ist, was zu dem linearen homogenen Gleichungssystem

$$
\sum_{j=1}^{p} c_{j}\left\{\int_{a}^{b} y_{j}(x) N w_{i}(x) d x-\mathfrak{R}^{N}\left(w_{i}\right) \mathfrak{x}\left(y_{j}\right)\right\}=0, \quad i=1, \ldots, p-1
$$

führt. Das sind $p-1$ Gleichungen für $p$ Unbekannte, die durch nicht sämtlich verschwindende $c_{i}$ erfüllt werden können. Wie K III, bestätigt man noch $(u, u)_{N}>0$ und $(u, u)_{M} \leqslant \lambda(u, u)_{N}$, womit (74) bewiesen ist.

Wählen wir nun $w_{i}(x)=y_{i}(x)$, haben wir zulässige Funktionen zu betrachten, für die $(u, u)_{N}>0$ und wegen (73)

$$
\left(u, y_{i}\right)_{N}=0, \quad i=1, \ldots, p-1
$$

gilt. Nach dem Rayleighschen Prinzip ist für dieses System $w_{i}(x)$

$$
m\left(y_{1}, \ldots, y_{p-1}\right)=\lambda_{p}
$$

und damit auch (76) bewiesen. Entsprechend kann (75), (77) gezeigt werden. Ebenso folgt bei Beachtung des Rayleighschen Prinzips die Behauptung für den eigentlich semidefiniten Fall.

Wir werden im folgenden noch eine andere Form des Courantschen Prinzips benötigen:

Satz 24. Das Courantsche Prinzip in Satz 23 behält für in $Z$ eigentlich definite Aufgaben Giiltigkeit, wenn unter $w_{t}(x)$ ein System $2 m$-mal stetig differenzierbarer Funktionen verstanden und an Stelle von (73) die Orthogonalitätsgleichung

$$
\int_{a}^{b} u(x) M w_{i}(x) d x-\Re^{M}\left(w_{i}\right) \mathfrak{x}(u)=0, \quad i=1, \ldots, p-1
$$

gefordert wird.

Beweis. Der Beweis von (74) und (75) kann wie in Satz 23 erfolgen. Um (76) zu zeigen, wählen wir wieder $w_{i}(x)=y_{i}(x)$ und haben alle zulässigen Funktionen zu betrachten, für die $(u, u)_{N}>0$ und

$$
\left(u, y_{i}\right)_{M}=0, \quad i=1, \ldots, p-1
$$


gilt. Aus

$$
\left(u, y_{i}\right)_{M}-\lambda_{i}\left(u, y_{i}\right)_{N}=0
$$

und $\lambda_{i} \neq 0$ bei eigentlich definiten Problemen folgt

$$
\left(u, y_{i}\right)_{N}=0, \quad i=1, \ldots, p-1
$$

und der Rest der Behauptung aus dem Rayleighschen Prinzip.

\section{Vergleichungssätze.}

SATZ 25. Die beiden natürlichen Eigenwertprobleme

$$
\left\{\begin{array}{l}
M y-\lambda N y=0 \\
W_{f}(y)=\mathbf{0} \\
R_{i}^{M}(y)=\lambda R_{j}^{N}(y)
\end{array}\right.
$$

$$
\left\{\begin{array}{l}
M y-\lambda^{*} N^{*} y=0, \\
W_{j}(y)=0, j=1, \ldots, k, \\
R_{j}^{M}(y)=\lambda^{*} R_{j}^{N^{*}}(y), j=1, \ldots, 2 m-k
\end{array}\right.
$$

seien eigentlich definit in $Z$. Beide Aufgaben besitzen dieselben wesentlichen Randbedingungen, dieselben Operatoren $M$ und Randoperatoren $R_{j}^{M}$. Wir bezeichnen die zweite natürliche Produktbildung der Aufgabe (80) mit $(u, u)_{N}^{*}$ und die Eigenwerte mit $\lambda_{i}^{*}$. Ist für jede zulässige Funktion

$$
(u, u)_{N}^{*} \geqslant(u, u)_{N}
$$

gilt für die Eigenwerte

$$
\lambda_{i}^{*} \leqslant \lambda_{1}, \quad i= \pm 1, \pm 2, \ldots,
$$

soweit sie existieren. Dabei sollen die Eigenwerte beider Aufgaben im Sinne von Satz 14 in einer Folge angeordnet sein.

Beweis. Es möge (79) positive Eigenwerte $\lambda_{1}$ besitzen. Dann existieren zulässige Funktionen $u(x)$ mit $(u, u)_{N}>0$ und wegen $(81) \mathrm{mu} B$ auch $(u, u)_{N}^{*}>0$ sein. Damit ist der Bereich aller Funktionen $\boldsymbol{u}(x)$ aus $Z$, für die $(u, u)_{N}>0$ ist, im Bereich aller Funktionen $u^{*}(x)$ aus $Z$ enthalten, die der Ungleichung $\left(u^{*}, u^{*}\right)_{N}^{*}>0$ genügen. Weiterhin ist

und

$$
R^{*}(u)=\frac{(u, u)_{M}}{(u, u)_{N}^{*}} \leqslant \frac{(u, u)_{M}}{(u, u)_{N}}=R(u)
$$

$$
\lambda_{1}^{*}=\operatorname{Min}_{\substack{u^{*} * Z \\\left(u^{*}, u^{*}\right)_{N}^{*}>0}} R^{*}\left(u^{*}\right) \leqslant \operatorname{Min}_{\substack{u \in Z \\\left(u, u_{N}>0\right.}} R^{*}(u) \leqslant \operatorname{Min}_{\substack{u \in Z \\(u, u)_{N}>0}} R(u)=\lambda_{1},
$$

also (82) bereits für $i=1$ bewiesen.

Für die höheren Eigenwerte ziehen wir das Courantsche Prinzip in der Form von Satz 24 heran. Es ist wieder der Bereich aller Funktionen $u(x)$ aus $Z$, die

$$
(u, u)_{N}>0, \quad\left(u, w_{i}\right)_{M}=0, \quad i=1, \ldots, p-1
$$

genügen, im Bereich der Funktionen $u^{*}(x)$ aus $Z$ enthalten, die

$$
\left(u^{*}, u^{*}\right)_{N}^{*}>0, \quad\left(u^{*}, w_{i}\right)_{M}=0, \quad i=1, \ldots, p-1
$$


erfüllen. Die Orthogonalitätsrelationen sind dabei für beide Aufgaben gleich, da sich (79), (80) nicht in $M$ und $R_{j}^{M}$ unterscheiden. Somit finden wir

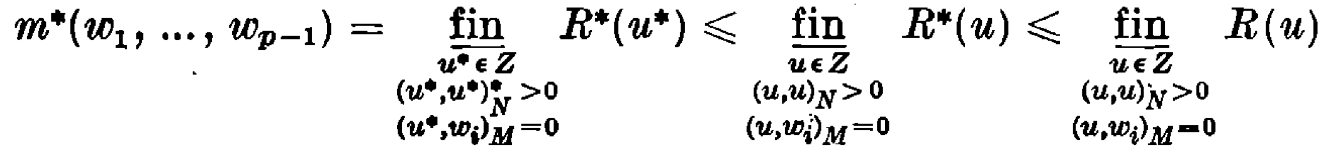

$$
\begin{aligned}
& =m\left(w_{1}, \ldots, w_{p-1}\right) \leqslant \lambda_{p} .
\end{aligned}
$$

Das gilt für beliebige $w_{\imath}(x)$, also kann $\lambda_{p}^{*} \leqslant \lambda_{p}$ gefolgert werden. Der Beweis für die negativen Eigenwerte ergibt sich entsprechend.

Folgerung: Ist bei den Aufgaben (79), (80) auch noch $N y=N^{*} y$, so daß sie sich nur in den Randoperatoren $R_{j}^{N}, R_{j}^{N^{*}}$ unterscheiden, reduziert sich die Voraussetzung (82) auf eine Annahme bezüglich der natürlichen Restteile

$$
\mathfrak{x}^{\prime}(u) \mathfrak{N}^{*} \mathfrak{x}(u) \geqslant \mathfrak{x}^{\prime}(u) \mathfrak{N} \boldsymbol{x}(u) .
$$

Damit ist insbesondere auch ein Vergleich der Aufgaben mit Kamkeschen Randbedingungen mit solchen möglich, bei denen natürliche Randbedingungen vorliegen.

SaTz 26. Betrachten wir zum Vergleich die in $Z$ eigentlich definiten Eigenwertprobleme (79) und

$$
\begin{gathered}
M^{*} y-\lambda * N y=0, \\
W_{\jmath}(y)=0, \quad j=1, \ldots, k, \\
R_{j}^{M^{*}}(y)=\lambda^{*} R_{j}^{N}(y), \quad i=1, \ldots, 2 m-k
\end{gathered}
$$

und gilt für jede zulässige Funktion

$$
(u, u)_{M}^{*} \geqslant(u, u)_{M},
$$

ist im Sinne von Satz 25

$$
\lambda_{1} \leqslant \lambda_{i}^{*}, \quad i= \pm 1, \pm 2, \ldots,
$$

soweit diese Eigenwerte existieren.

Beweis. Es ist offenbar $R^{*}(u) \geqslant R(u)$ und der Bereich der $u(x)$, $u^{*}(x)$ in Sinne von Satz 25 gleich. Wie in Satz 25, folgt die Behauptung, wenn man sich auf das Courantsche Prinzip von Satz 23 bezieht.

Somit ist es gelungen, das von Kamke entwickelte Vorgehen auf natürliche Eigenwertprobleme auszudehnen und sich von der einschneidenden Voraussetzung zu befreien, daß in den restlichen Randbedingungen der Eigenparameter nicht auftreten darf. Die Ausdehnung dieser Gedankengänge auf das Ritz-Galerkinsche Verfahren, die Entwicklung nach Eigenfunktionen und das Iterationsverfahren. soll in besonderen Mitteilungen erfolgen. 


\section{Literaturverzeichnis}

[1] L. Collatz, Eigenwertaufgaben mit technischen Anwendungen, Leipzig 1949.

[2] W. Dück, Ein Entwicklungssatz bei iinearen, selbstadjungierten, volldefiniten Eigenwertproblemen gewöhnlicher Differentialgleichungen, Z. angew. Math. Mech. 39 (1959), S. 358-363.

[3] - Bemerkungen zum Minimalprinzip von Kamke, Mitteilungen d. Math. Gesellschaft d. DDR (965), 1. Heft.

[4] - Ein Entwicklungssatz für zulässige Funktionen, Erscheint demnächst.

[5] E. Kamke, Über die definiten selbstadjungierten Eigenwertaufgaben bei gewöhnlichen linearen Differentialgleichungen II, Math. Zeitschr. 46 (1940), S. 231-250.

[6] - Über die definiten selbstadjungierten Eigenwertaufgaben bei gewöhnlichen linearen Differentialgleichungen III, Math. Zeitschr. 46 (1940), S. 251-286.

[7] - Über die definiten selbstadjungierten Eigenwertaufgaben bei gewōhnlichen linearen Differentialgleichungen IV, Math. Zeitschr. 48 (1942/43), S. 67-100.

[8] N. J. Lehmann, Eine Integraldarstellung für selbstadjungierte Randwertaufgaben (einschließlich einer Theorie der natürlichen Eigenwertprobleme), Math. Nachr. 14 (1955), S. 129-156.

[9] - Das inhomogene natürliche Randwertproblem und Fehlerabschätzungen für Näherungstosungen, Num. Math. 3 (1961), S. 1-29.

[10] E. Stiefel und H. Ziegler, Natürliche Eigenwertprobleme, Z. angew. Math. Phys. 1 (1950), S. 111-138.

Reçu par la Rédaction le 19.12.1963 